\title{
Colors of 2625 Quasars at $0<z<5$ Measured in the Sloan Digital Sky Survey Photometric System
}

\section{Citation}

Richards, Gordon T., Xiaohui Fan, Donald P. Schneider, Daniel E. Vanden Berk, Michael A. Strauss, Donald G. York, John E. Anderson, Jr., et al. 2001. "Colors of 2625 Quasars at $0<z<5$ Measured in the Sloan Digital Sky Survey Photometric System." The Astronomical Journal 121 (5) (May): 2308-2330. doi:10.1086/320392.

\section{Published Version}

doi:10.1086/320392

\section{Permanent link}

http://nrs.harvard.edu/urn-3:HUL.InstRepos:33461994

\section{Terms of Use}

This article was downloaded from Harvard University's DASH repository, and is made available under the terms and conditions applicable to Other Posted Material, as set forth at http:// nrs.harvard.edu/urn-3:HUL.InstRepos:dash.current.terms-of-use\#LAA

\section{Share Your Story}

The Harvard community has made this article openly available.

Please share how this access benefits you. Submit a story.

\section{Accessibility}


COLORS OF 2625 QUASARS AT $0<z<5$ MEASURED IN THE SLOAN DIGITAL SKY SURVEY PHOTOMETRIC SYSTEM ${ }^{1}$

Gordon T. Richards, ${ }^{2,3}$ Xiaohui Fan, ${ }^{4,5}$ Donald P. Schneider, ${ }^{2}$ Daniel E. Vanden Berk, ${ }^{6}$ Michael A. Strauss, ${ }^{4}$ Donald G. York, ${ }^{3,7}$ John E. ANDERson, JR., ${ }^{6}$ Scott F. ANDerson, ${ }^{8}$ JAmes AnNis, ${ }^{6}$ Neta A. BahCall, ${ }^{4}$ Mariangela Bernardi, ${ }^{3}$ John W. Briggs, ${ }^{3}$ J. Brinkmann,${ }^{9}$ Robert Brunner, ${ }^{10}$ Scott Burles, ${ }^{3,6}$ Larry Carey, ${ }^{8}$ Francisco J. Castander, ${ }^{3,11}$ A. J. ConNolly, ${ }^{12}$ J. H. Crocker,${ }^{13}$ István Csabai, ${ }^{13,14}$ Mamoru DoI, ${ }^{15}$ Douglas Finkbeiner, ${ }^{16}$ Scott D. Friedman, ${ }^{13}$ Joshua A. Frieman, ${ }^{3,6}$ Masataka Fukugita, ${ }^{17}$ James E. GunN, ${ }^{4}$ Robert B. HindSLEy,${ }^{18}$ ŽelJKo IVEZIĆ, ${ }^{4}$ STEPHEN Kent,${ }^{3,6}$ G. R. KNAPP,${ }^{4}$ D. Q. LAMb, ${ }^{3}$ R. FrenCh LeGER, ${ }^{8}$ Daniel C. Long, ${ }^{9}$ Jon Loveday ${ }^{19}$ Robert H. Lupton ${ }^{4}{ }^{4}$ Timothy A. MCKay, ${ }^{20}$ Avery Meiksin, ${ }^{21}$ Aronne Merrelli, ${ }^{10,22}$ Jeffrey A. Munn, ${ }^{23}$ Heidi Jo Newberg, ${ }^{24}$ Matt Newcomb,${ }^{22}$ R. C. Nichol, ${ }^{22}$ Russell Owen, ${ }^{8}$ JefFrey R. Pier, ${ }^{23}$ Adrian Pope,${ }^{13,22}$ Michael W. Richmond, ${ }^{25}$ Constance M. Rockosi, ${ }^{3}$ David J. Schlegel, ${ }^{4}$ Walter A. Siegmund, ${ }^{8}$ StePhen Smee, ${ }^{13,26}$ Yehuda Snir, ${ }^{22}$ Chris Stoughton, ${ }^{6}$ Christopher Stubbs, ${ }^{8}$ Mark SubbaRao, ${ }^{3}$ Alexander S. Szalay, ${ }^{13}$ Gyula P. Szokoly,${ }^{27}$ Christy Tremonti, ${ }^{13}$ Alan Uomoto, ${ }^{13}$ Patrick WadDEll ${ }^{8}$ Brian YanNy, ${ }^{6}$ AND Wei Zheng ${ }^{13}$

Received 2000 November 6; accepted 2000 December 11

\section{ABSTRACT}

We present an empirical investigation of the colors of quasars in the Sloan Digital Sky Survey (SDSS) photometric system. The sample studied includes 2625 quasars with SDSS photometry: 1759 quasars found during SDSS spectroscopic commissioning and SDSS follow-up observations on other telescopes, 50 matches to FIRST quasars, 573 matches to quasars from the NASA Extragalactic Database, and 243 quasars from two or more of these sources. The quasars are distributed in a 2.5 wide stripe centered on the celestial equator covering $\sim 529 \mathrm{deg}^{2}$. Positions (accurate to 0 "'2) and SDSS magnitudes are given for the 898 quasars known prior to SDSS spectroscopic commissioning. New SDSS quasars, which range in brightness from $i^{*}=15.39$ to the photometric magnitude limit of the survey, represent an increase of over $200 \%$ in the number of known quasars in this area of the sky. The ensemble average of the observed colors of quasars in the SDSS passbands are well represented by a power-law continuum with $\alpha_{v}=-0.5\left(f_{v} \propto v^{\alpha}\right)$ and are close to those predicted by previous simulations. However, the contributions of the "small blue (or 23000 ) bump" and other strong emission lines have a significant effect upon the colors. The color-redshift relation exhibits considerable structure, which may be of use in determining photometric redshifts for quasars from their colors alone. The range of colors at a given redshift can generally be accounted for by a range in the optical spectral index with a distribution $\alpha_{v}=-0.5 \pm 0.65$

\footnotetext{
${ }^{1}$ Based on observations obtained with the Sloan Digital Sky Survey; with the Apache Point Observatory $3.5 \mathrm{~m}$ telescope, which is owned and operated by the Astrophysics Research Consortium; with the Hobby-Eberly Telescope, which is a joint project of the University of Texas at Austin, the Pennsylvania State University, Stanford University, Ludwig-Maximillians-Universität München, and Georg-August-Universität Göttingen; with the $2.1 \mathrm{~m}$ telescope at Kitt Peak National Observatory; and at the W. M. Keck Observatory, which is operated as a scientific partnership among the California Institute of Technology, the University of California, and NASA and was made possible by the generous financial support of the W. M. Keck Foundation.

${ }^{2}$ Department of Astronomy and Astrophysics, Pennsylvania State University, University Park, PA 16802.

${ }^{3}$ Department of Astronomy and Astrophysics, University of Chicago, 5640 South Ellis Avenue, Chicago, IL 60637.

${ }^{4}$ Princeton University Observatory, Princeton, NJ 08544.

${ }_{6}^{5}$ Institute for Advanced Study, Olden Lane, Princeton, NJ 08540.

${ }^{6}$ Fermi National Accelerator Laboratory, P.O. Box 500, Batavia, IL 60510.

${ }^{7}$ Enrico Fermi Institute, University of Chicago, 5640 South Ellis Avenue, Chicago, IL 60637.

8 Department of Astronomy, University of Washington, Box 351580, Seattle, WA 98195.

9 Apache Point Observatory, P.O. Box 59, Sunspot, NM 88349-0059.

${ }^{10}$ Department of Astronomy, California Institute of Technology, Pasadena, CA 91125.

${ }^{11}$ Observatoire Midi Pyrénées, 14 avenue Edouard Belin, Toulouse, F-31400, France.

12 Department of Physics and Astronomy, University of Pittsburgh, Pittsburgh, PA 15260.

13 Department of Physics and Astronomy, Johns Hopkins University, 3701 San Martin Drive, Baltimore, MD 21218.

14 Department of Physics of Complex Systems, Eötvös Loránd University, Pázmány Péter sétány 1/A, H-1117 Budapest, Hungary.

${ }^{15}$ Department of Astronomy and Research Center for the Early Universe, School of Science, University of Tokyo, Hongo, Bunkyo, Tokyo, 113-0033, Japan.

${ }^{16}$ Departments of Physics and Astronomy, 601 Campbell Hall, University of California, Berkeley, CA 94720.

17 University of Tokyo, Institute for Cosmic-Ray Research, Kashiwa, 2778582, Japan.

18 Remote Sensing Division, Code 7215, Naval Research Laboratory, 4555 Overlook Avenue SW, Washington, DC 20375.

${ }^{19}$ Astronomy Centre, University of Sussex, Falmer, Brighton BN1 9QJ, England, UK.

${ }^{20}$ Department of Physics, University of Michigan, 500 East University Avenue, Ann Arbor, MI 48109.

${ }^{21}$ Royal Observatory, Edinburgh, EH9 3HJ, England, UK.

22 Department of Physics, Carnegie Mellon University, 5000 Forbes Avenue, Pittsburgh, PA-15232.

${ }^{23}$ U.S. Naval Observatory, Flagstaff Station, P.O. Box 1149, Flagstaff, AZ 86002-1149.

${ }^{24}$ Department of Physics, Rensselaer Polytechnic Institute, SC1C25, Troy, NY 12180.

${ }^{25}$ Department of Physics, Rochester Institute of Technology, 85 Lomb Memorial Drive, Rochester, NY 14623-5603.

${ }^{26}$ Department of Astronomy, University of Maryland, College Park, MD 20742-2421.

${ }^{27}$ Astrophysikalisches Institut Potsdam, An der Sternwarte 16, D-14482 Potsdam, Germany.
} 
( $95 \%$ confidence), but there is a red tail in the distribution. This tail may be a sign of internal reddening, especially since fainter objects at a given redshift tend to exhibit redder colors than the average. Finally, we show that there is a continuum of properties between quasars and Seyfert galaxies, and we test the validity of the traditional dividing line $\left(M_{B}=-23\right)$ between the two classes of active galactic nuclei.

Key words: catalogs - quasars: general — surveys

On-line material: machine-readable table

\section{INTRODUCTION}

Quasar identification in multicolor surveys relies on the fact that the spectral energy distributions of stars and quasars produce different colors in broadband photometric systems. At low redshift $(z \leq 2.2)$, the lack of a Balmer jump in quasars separates them from hot stars; at higher redshifts the presence of the strong Ly $\alpha$ emission line and absorption by the Ly $\alpha$ forest cause the broadband colors of quasars to become increasingly redder with redshift (Sandage 1965; Green et al. 1986; Warren et al. 1991a; Fan 1999). Historically, large area surveys have been based on photographic plates; the limited photometric accuracy of this technique $(\sim 0.1 \mathrm{mag})$ has been one of the primary limitations of the effectiveness of their quasar selection.

The Sloan Digital Sky Survey (SDSS; York et al. 2000) will survey approximately one-quarter of the celestial sphere in five broad bands spanning the optical region of the spectrum. The expected photometric errors in each filter are less than $5 \%$ for objects brighter than $19^{\text {th }}$ magnitude; this should allow identification of approximately 100,000 quasars. In this paper we study the photometric properties in the SDSS system of over 2600 quasars in a stripe 2.5 wide centered on the celestial equator; the total area covered is $\sim 529 \mathrm{deg}^{2}$. Over 1700 of these objects were discovered in the past year and are examined here for the first time.

This paper acts as an empirical companion to Fan (1999), which presented theoretical and simulated colors of quasars and other point sources in the SDSS system. Although the SDSS photometric system is still uncertain at the few percent level, the photometry is better than has ever been accomplished previously for a large area sample of quasars. With the SDSS photometry, we can detect features in the color-redshift distribution that are smaller than the typical error in the colors of quasars from photographic surveys. Using this information, we examine a number of longstanding issues in quasar science.

Section 2 of the paper describes the observations, and in $\S 3$ we describe the catalogs that were used to construct the database for this study. In $\S 4$ we examine the observed colors of quasars and compare them with the values expected from simulations of their spectral energy distributions, and in $\S 5$ we discuss how these results will impact a number of issues, including quasar target selection in the SDSS. Finally, § 6 presents our conclusions.

\section{SDSS IMAGING}

The Sloan Digital Sky Survey (York et al. 2000) will provide accurate photometry and spectroscopy for over $10,000 \mathrm{deg}^{2}$ of sky. The optical magnitudes of detected objects are measured nearly simultaneously through five broadband filters $\left(u^{\prime}, g^{\prime}, r^{\prime}, i^{\prime}\right.$, and $z^{\prime}$; Fukugita et al. 1996) with nominal effective wavelengths of 3540, 4760, 6280, 7690 , and $9250 \AA$, complete to limiting point-source magnitudes of 22.3, 22.6, 22.7, 22.4, and 20.5, respectively (signalto-noise ratio 5:1). Because the definition of the photometric system is not yet finalized, measured magnitudes are quoted using asterisks $\left(u^{*}, g^{*}, r^{*}, i^{*}\right.$, and $\left.z^{*}\right)$ to represent preliminary photometry; the filters themselves are referred to as $u^{\prime}, g^{\prime}, r^{\prime}, i^{\prime}$, and $z^{\prime}$. SDSS magnitudes are based on the AB magnitude system (Oke \& Gunn 1983) and are given as asinh magnitudes (Lupton et al. 1999), which have nice properties at low flux limits. For objects fainter than the $5 \sigma$ flux limit given above, the asinh magnitude will deviate from normal magnitudes. The analysis in this paper should not be affected by these differences; however, some of the high-redshift quasars have $u^{\prime}$ and $g^{\prime}$ magnitudes fainter than these limits.

The survey is being done with a dedicated $2.5 \mathrm{~m}$ telescope (Siegmund et al. 2001). The telescope has a wide, wellcorrected field and is equipped with a large mosaic CCD camera (Gunn et al. 1998) and a pair of fiber-fed spectrographs (Uomoto et al. 2001a). The camera utilizes 30 $2048 \times 2048$ CCDs which take the data in drift-scanning (time-delay-and-integrate, or TDI) mode with a total integration time of $54.1 \mathrm{~s}$ per filter. The imaging data are obtained using the data acquisition system (Petravick et al. 1994; Annis et al. 2001) at the Apache Point Observatory (APO) and are automatically processed through a set of software pipelines (Kent et al. 2001). The photometric pipeline (Lupton et al. 2001) reduces the imaging data, measuring positions, magnitudes, and shape parameters for all detected objects. The photometric pipeline uses information from the astrometric pipeline (Pier et al. 2001) and the photometric calibration telescope (PT; Smith et al. 2001; Uomoto et al. 2001b; Tucker et al. 2001). After final photometric calibration of the data, the outputs, together with all the observing and processing information, are loaded into the operational database (Yanny et al. 2001). The final parameters are stored in an object-oriented searchable database (SX; Szalay et al. 2001). For an explanation of SDSS technical terms used in the text, please refer to York et al. (2000).

Preliminary analysis shows that there are differences between the real SDSS transmission curves and those shown by Fukugita et al. (1996); see Fan et al. (2001), Appendix A, for a discussion. These differences are believed to be due to the fact that the filters are in vacuo and are not exposed to the air. For a quasar with a typical power-law spectrum of $f_{v} \propto v^{-0.5}$ we find that the effective wavelengths of the actual $2.5 \mathrm{~m}$ filters are $3651,4679,6175,7494$, and $8873 \AA$ instead of $3544,4770,6231,7625$, and $9135 \AA$, respectively, for $u^{\prime}, g^{\prime}, r^{\prime}, i^{\prime}$, and $z^{\prime}$. (Since the spectral energy distribution of a quasar is different from the standard stars used to define the SDSS photometric system, the latter effective wavelengths of the passbands are different from those quoted earlier.) These offsets are quite large, but they produce only small effects on the measured colors of the objects discussed herein $(\sim 0.005 \mathrm{mag})$. The most current transmission curves are used in all of the analysis throughout the paper. More details on the transmission curves of the $2.5 \mathrm{~m}$ camera will be shown in a future paper (Doi et al. 2001). 
TABLE 1

SUMMARY OF OBSERVATIONS

\begin{tabular}{ccccc}
\hline \hline Run & UT Date & Strip & $\begin{array}{c}\text { R.A. Range } \\
(\mathrm{J} 2000.0)\end{array}$ & $\begin{array}{c}\text { Seeing } \\
(\operatorname{arcsec})\end{array}$ \\
\hline $94 \ldots \ldots$. & 1998 Sep 19 & North & $2216-0349$ & 1.5 \\
$125 \ldots \ldots$ & 1998 Sep 25 & South & $2318-0517$ & 1.5 \\
$752 \ldots \ldots$. & 1999 Mar 21 & South & $0821-1541$ & $1.5-1.8$ \\
$756 \ldots \ldots$. & 1999 Mar 22 & North & $0821-1634$ & $1.2-1.5$ \\
\hline
\end{tabular}

Data from the imaging camera on the $2.5 \mathrm{~m}$ are calibrated against patches of the sky observed by the PT. The PT also observes standard stars in order to determine nightly extinction coefficients. While the quality of the current SDSS photometric data is more than adequate for our purposes, the final calibration of the SDSS system is not yet in place, due in part to the SDSS transmission curve uncertainties discussed above. However, the quality of the data in hand suggests that any changes to the quasar colorcolor and color-redshifts relations will be relatively minor and should not have a significant impact on the results presented herein.

The SDSS data analyzed herein include the photometric catalog from the four best equatorial scans that were taken between 1998 September 19 and 1999 March 22, which include data "runs" 94, 125, 752, and 756, as described in Table 1. These runs were observed and processed as part of the commissioning phase of the SDSS; they were acquired with the telescope parked on the meridian while pointed at the celestial equator. The density of previously known quasars is highest on the celestial equator, making these data particularly appropriate for this study. Runs 94 and 125 are from the south Galactic cap, whereas runs 752 and 756 are from the north Galactic cap. A fraction of the data from these runs have been re-reduced. The mean difference between the old magnitudes and the new magnitudes is $(0.043,0.008,0.007,0.006,0.006)$ in $\left(u^{*}, g^{*}, r^{*}, i^{*}, z^{*}\right)$ coordinates. Since these differences are negligible, we will use the "old" magnitudes throughout for the sake of consistency.

\section{QUASAR CATALOG}

In order to study the colors of quasars in the SDSS photometric system, we first generated a (full-sky) master catalog of all known quasars including the newly discovered quasars from the SDSS. We then matched the equatorial SDSS photometric catalog to the equatorial regions of the master catalog. The master quasar catalog used in this study includes quasars from four distinct sources. These four subcatalogs of quasars were combined to form a larger combined catalog. The subcatalogs are described in the following sections.

\subsection{NED}

One-third of the quasars studied herein are previously known quasars that have been cataloged by the NASA Extragalactic Database (NED). ${ }^{28}$ Our NED subcatalog includes all of the 12,987 objects that NED classified as quasars with known redshifts as of 2000 June 22. Many of

\footnotetext{
${ }^{28}$ The NASA/IPAC Extragalactic Database (NED) is operated by the Jet Propulsion Laboratory, California Institute of Technology, under contract with the National Aeronautics and Space Administration.
}

these objects will also appear in the other subcatalogs discussed later.

The NED catalog is a compilation of results from the literature. Any bias in the original selection of these objects will also be manifest in this sample. One of the most important selection effects is the definition of what constitutes a quasar. Typically, quasars are defined to be active galactic nuclei (AGNs) brighter than $M_{B}=-23\left(H_{0}=50 \mathrm{~km} \mathrm{~s}^{-1}\right.$ $\mathrm{Mpc}^{-1}, q_{0}=0.5$ ); however, Seyfert galaxies, the fainter cousins of quasars, are often included in compilations of quasars. Such objects from NED are included in our combined catalog for sake of completeness even though their colors may be contaminated by their host galaxy. See $\S 5.5$ for more on this subject.

The primary input to the NED quasar catalog is the catalog produced by Hewitt \& Burbidge (1989), hereafter HB89, which includes over 3500 quasars. As with the NED catalog, HB89 is itself a compilation from the literature. The largest uniform sample of quasars $(\sim 1000)$ in NED comes from the Large Bright Quasar Survey (LBQS; Hewett et al. 1995). Since LBQS quasars were selected from objective prism plates, the LBQS catalog may include quasars with stronger than average emission lines. Other optical quasar surveys that contribute significantly to the catalog of known quasars include the AAT Survey (Boyle et al. 1990), the CFHT Survey (Crampton et al. 1988), the Second Byurakan Survey (SBS; Stepanian et al. 1993), and the Palomar Grism Surveys (Schneider et al. 1994; Schneider et al. 1999). Not all of these surveys are color-selected surveys; each has its own selection effects. The AAT survey was an ultraviolet excess (UVX) survey and is biased toward $z<2.2$. The CFHT survey used a blue "grens" to do slitless spectroscopy and was sensitive to $z<3.3$. The SBS survey was an objective prism survey, whereas the Palomar Grism Surveys used a grism for candidate selection and slit spectroscopy for follow-up observations. Many of the radio-detected quasars in NED come from Véron-Cetty \& Véron (1996), which, like HB89, is a compilation based on a variety of sources. The brightest of these objects are 3C sources (Spinrad et al. 1985), which are brighter than 9 Jy at 178 MHz.

\subsection{FIRST}

We have attempted to recover, in the SDSS data, quasars detected by the VLA FIRST Survey (Becker et al. 1995), in particular those discovered by the FIRST Bright Quasar Survey (FBQS; Gregg et al. 1996; White et al. 2000; Becker et al. 2001) and the FIRST Faint Quasar Survey (FFQS; Becker et al. 1998). Many of the FBQS quasars also appear in the NED catalog; however, the FBQS is an ongoing survey, so we have included these FIRST quasars explicitly. Although the SDSS will be selecting FIRST sources as quasar candidates, it is valuable to include in our sample those FIRST objects that have already been confirmed as quasars. As with the radio-selected quasars from NED, FIRST quasars are valuable because they have different selection biases than optically selected quasars. In particular, they can be used to determine the fraction of quasars embedded in the stellar locus and to analyze the effects of reddening and extinction upon quasar target selection.

\subsection{SDSS Spectroscopic Commissioning}

The largest part of the sample comes from spectroscopic confirmation of quasar candidates from SDSS spectro- 
scopic commissioning (Uomoto et al. 2001a). The SDSS uses two double fiber-fed spectrographs; each has separate red and blue cameras, with a dichroic splitting the light at $\sim 6000 \AA$. The fibers are hand-plugged into an aluminum plate (640 fibers per plate); the correspondence between hole number and fiber number is determined by an automated plate mapper. The entire fiber, plate, and slit assembly is modular and is removed and replaced for each spectroscopic exposure.

The fibers subtend $3^{\prime \prime}$ at the focal plane. The resulting spectra cover the range from 3800 to $9200 \AA$ with a resolution of roughly 1800 over 4096 pixels. Typical exposure times are 45 minutes to an hour, broken into single exposures of 15 minutes each. Reduction on the mountain confirms that the signal-to-noise ratio of the faintest objects meets survey specifications. The wavelength calibration is appreciably better than the specifications of $0.1 \AA$. The flux calibration is currently only approximate and is carried out with observations of $\mathrm{F}$ subdwarfs. The spectrographs are very high throughput, approaching $30 \%$ in the red; the resulting spectra have quite high signal-to-noise ratio, of order 4 pixel $^{-1}$ for objects as faint as $r^{\prime}=20$.

Quasar candidates for spectroscopy were selected by a preliminary version of the SDSS Quasar Target Selection Algorithm (Newberg et al. 2001; Richards et al. 2001), which is primarily a color-selection algorithm. The primary selection criterion is that objects be outliers from the stellar locus, which means that quasars with unusual colors will be targeted. Since one of the goals of the commissioning phase of this project is to define this algorithm, the quasars selected with the current algorithm cannot be considered representative of the final SDSS quasar sample, or in any way to constitute a complete sample. In particular, these are colorselected objects selected with color cuts that are not yet final (moreover, the color cuts have changed with time), and the quasars do not cover a uniform area on the sky. Nevertheless, they are extremely useful for increasing our knowledge of the colors of quasars.

Quasar candidates were observed with the SDSS fiber spectrographs, and the data were reduced with the spectroscopic pipeline (Frieman et al. 2001). Each of the quasar identifications and redshifts were determined by visual inspection by one of us (D. E. V. B.), since the automated reduction pipelines are not yet complete. The redshifts and photometry of these 1677 objects are not included in Table 2 but will appear in 2001 as part of a public SDSS data release. A sample of 10 SDSS quasar spectra with $0<z<5$ are presented in Figure 1, in order to give the reader an idea of the quality of the spectra.

\subsection{SDSS Follow-up}

The remainder of the sample includes 112 quasars discovered during the course of SDSS follow-up observations using the ARC $3.5 \mathrm{~m}$ telescope at Apache Point Observatory, the Hobby-Eberly Telescope at McDonald Observatory, and the W. M. Keck Observatory. These observations are part of a number of follow-up projects that are underway, mostly in search of high- $z$ quasars. Quasar candidates are selected by color and confirmed spectroscopically on these other telescopes in order to confirm objects that the SDSS spectrographs might not observe (e.g., because of faintness, fiber spacing constraints, etc.) or that the SDSS has simply not taken a spectrum of yet. Those SDSS followup objects used in this study have been published by Fan et al. (1999), Fan et al. (2000), Schneider et al. (2000), Zheng et al. (2000), Fan et al. (2001), and Schneider et al. (2001). The redshift and color distribution of this sample of quasars is summarized in the last of these references.

Nearly all of the $z \geq 3.6$ quasars in our sample are from these SDSS follow-up observations (which cover a larger area of sky than the SDSS spectroscopic commissioning plates). The selection of these quasars introduces a significant feature in the redshift distribution of our sample: certain regions $(3<z<3.6)$ of color space are apparently underrepresented because of the way that high-redshift follow-up targets were selected. The selection criteria for the $z \geq 3.6$ quasars are presented by Fan et al. (2001) and references therein.

\subsection{Combined Catalog}

The combination of these subcatalogs results in a total of 16,446 quasars covering the entire sky, a fraction of which are in the region covered by the SDSS commissioning data from the celestial equator. The distribution of quasars is as follows: 12,987 are from the NASA Extragalactic Database (NED), 941 are FIRST quasars, and 2518 were discovered or recovered as part of the SDSS. The quasars found using SDSS data are from both the spectroscopic commissioning data (2406 quasars) and from SDSS follow-up observations using other telescopes (112 quasars).

In order to create a combined catalog that includes each quasar only once, the NED, FIRST, and SDSS subcatalogs were compared with each other. Quasars in more than one catalog whose positions agreed to $5^{\prime \prime}$ or better, whose redshifts agreed to $\Delta z=0.1$ or better, and whose differential magnitude differed by less than 3.5 mag were considered to be matches. In all, 508 quasars were found in more than one catalog of quasars, resulting in a total of 15,938 unique quasars in the full-sky catalog.

\subsection{SDSS Matches to the Combined Catalog}

In four SDSS equatorial scans studied, 2625 previously known or recently discovered quasars from the combined full-sky catalog of 15,938 quasars are found in the SDSS database. Quasars from the combined catalog were considered to be matches to the SDSS database if the positions of the quasars and the SDSS objects agreed to better than 3 , and the agreement between the cataloged magnitudes and the SDSS magnitudes was reasonable (rms of the difference between the predicted and observed $g^{\prime}$ and $r^{\prime}$ magnitudes less than $2 \mathrm{mag}$, where the predicted SDSS magnitudes have been computed by transforming the published magnitude to the SDSS system assuming a spectral index of $\alpha_{v}=-0.5$ ). The SDSS astrometry is good to 0 0.2 or better per coordinate. See Finlator et al. (2000), Figure 2, for a comparison of the SDSS astrometry that demonstrates this level of accuracy. An additional 235 known quasars had potential matches, but the positions disagreed by more than $3^{\prime \prime}$. Many of these matches with large positional discrepancies may be correct, but in order to avoid contamination of the empirical colors studies herein, we do not include these in our analysis.

Of these 2625 quasars, 801 are from NED (573 unique), 92 are from FIRST (50 unique), and 1983 quasars are from SDSS data (1759 unique). A total of 243 quasars were found in more than one of the above catalogs. There are more NED quasars in the area covered by these four runs; however, only these 801 had NED coordinates and photo- 


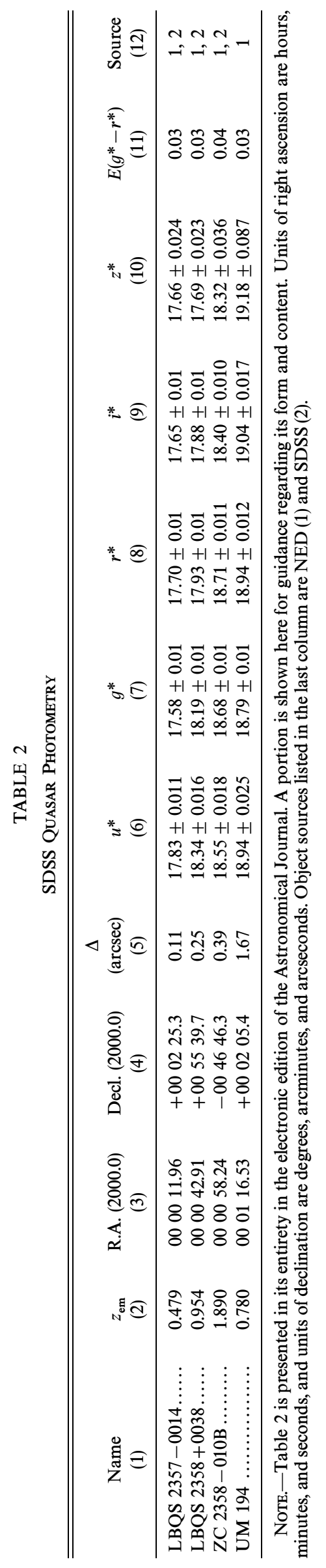



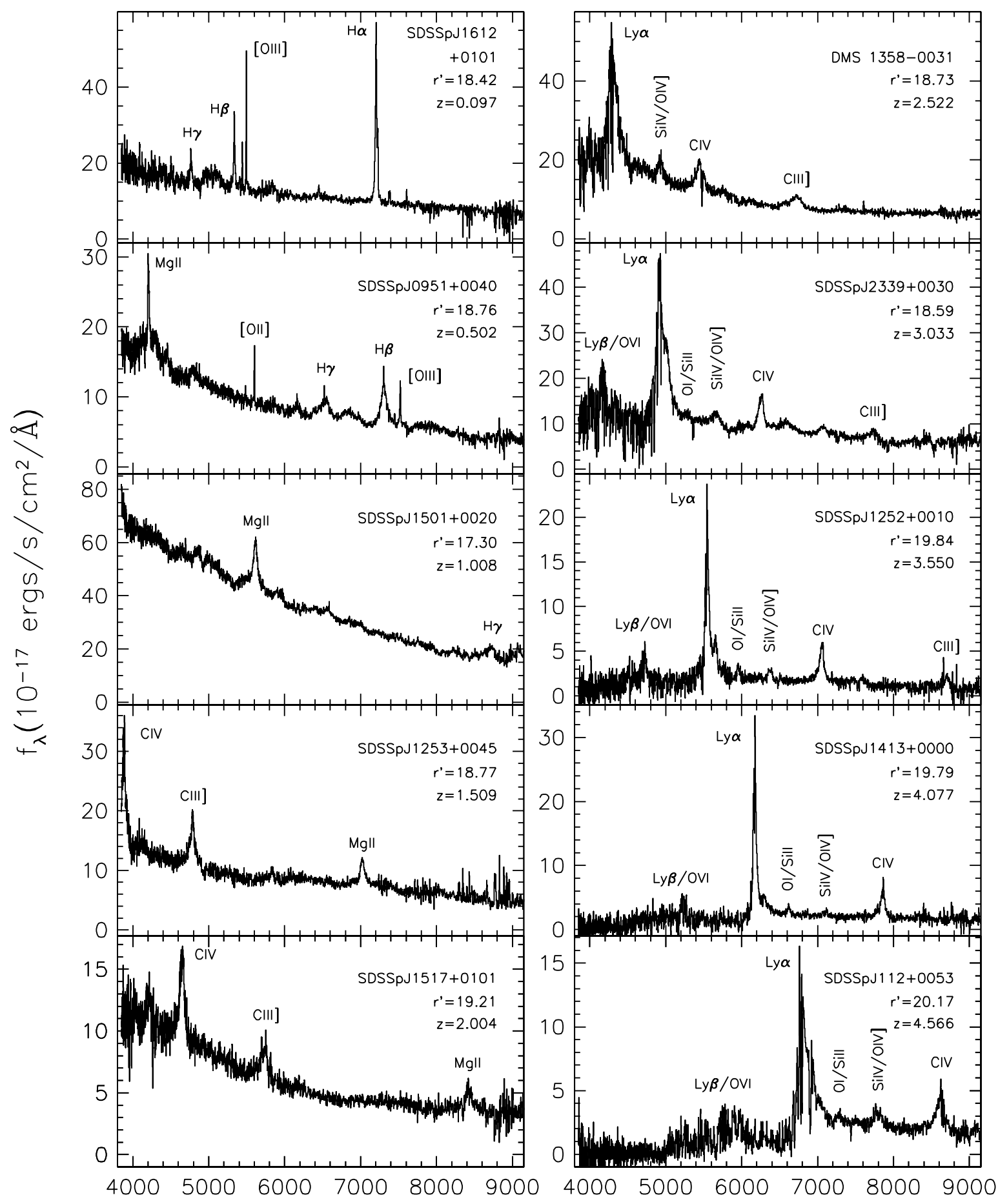

Wavelength $(\AA)$

FIG. 1.-Sample SDSS quasars. These quasars are representative of the quasars found in the SDSS sample from $z \sim 0$ to $z \sim 5$.

metry accurate enough to ensure that the matches to SDSS objects are correct. A more detailed (and labor intensive!) analysis would allow for the matching of additional known quasars and for the correction of their published coordinates. A histogram of the redshift distribution of all 2625 quasars is given in Figure 2. There is a noticeable lack of quasars between $z=2.5$ and $z=3.6$, since the density of quasars at $z \geq 2.5$ is declining, and the follow-up observations specifically target quasars at $z \geq 3$.6. The new SDSS quasars represent over a factor of two increase in the density of known quasars on the celestial equator.

The difference between the input magnitude (typically, but not always, $B_{J}$ ) and the measured $g^{*}$ magnitude for the NED and FIRST sources are plotted as a function of $g^{*}$ in Figure 3. This plot enables us to check the accuracy of the cataloged magnitudes and to look for variability (e.g., Francis 1996). Photometry and astrometry for all previously published quasars (including SDSS quasars dis- 


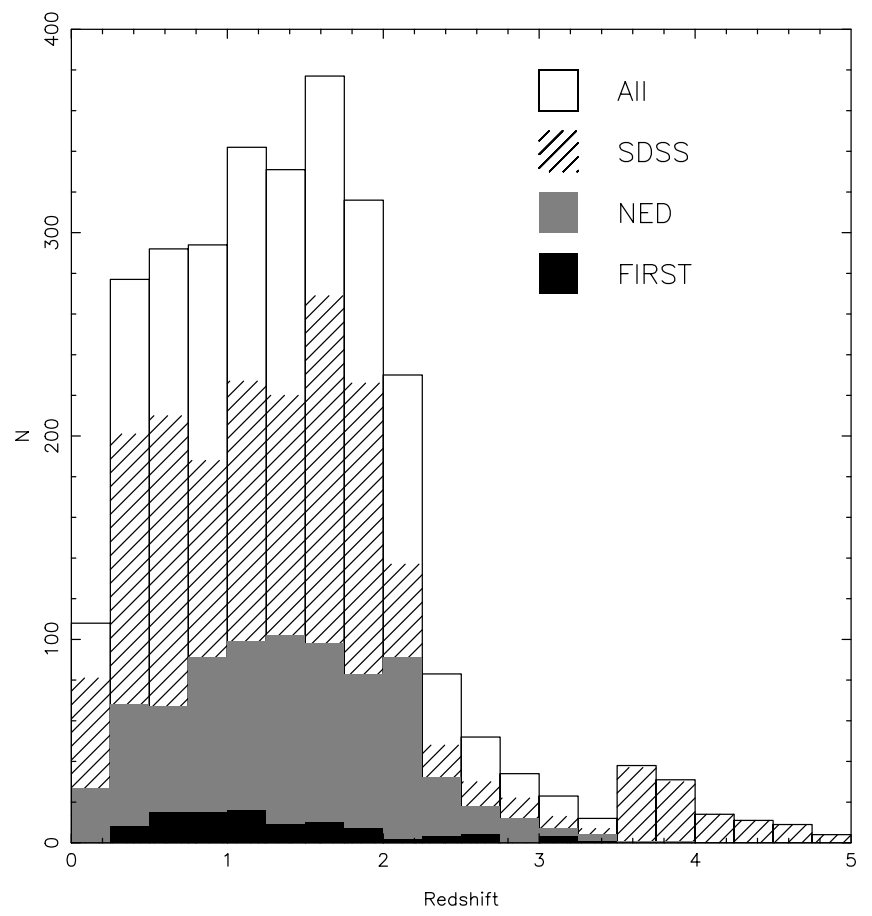

FIG. 2.- Redshift distribution for 801 NED quasars, 92 FIRST quasars, and 1983 SDSS quasars. Some quasars appear in more than one sample. There are 2625 quasars in total.

covered during follow-up observations) is presented in Table 2. Data on the 1677 new SDSS quasars will be published separately as part of an official SDSS data release. Data on the 50 FIRST equatorial quasars was kindly provided in advance of publication by R. Becker and will be presented by Becker et al. (2001). The columns in

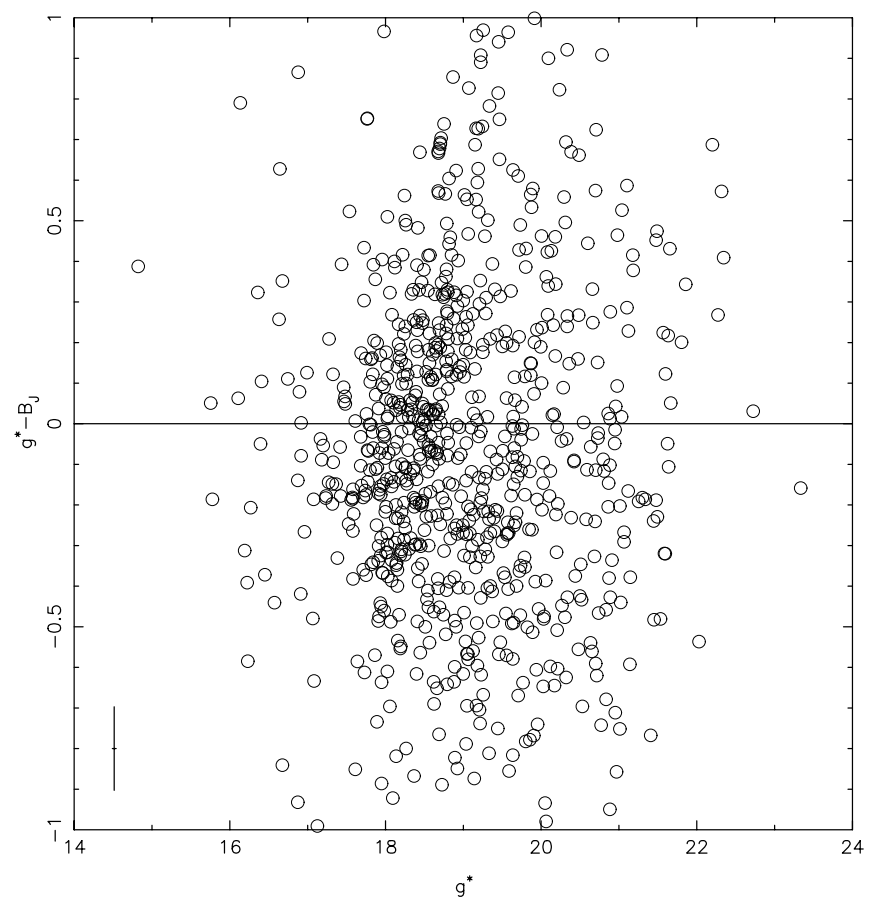

FIG. 3.-Magnitude difference vs. magnitude for 867 NED and FIRST quasars. For NED quasars the cataloged magnitude is typically, but not always, $B_{J}$; for FIRST quasars $B_{J}$ is replaced by $O$. The cross in the bottom left-hand corner shows a $3 \%$ error in $g^{*}$ and the error in $g^{*}-B_{J}$ given a $10 \%$ error in $B_{J}$.
Table 2 are as follows: (1) is the name of the quasar. Column (2) is the redshift. The right ascension and declination as measured by the SDSS are given as J2000.0 coordinates in columns (3) and (4). The difference between the SDSS position and the cataloged position is given in column (5) in units of arcseconds. Columns (6) through (10) give the measured magnitudes and errors in the five SDSS passbands. Errors are photometric errors only and do not include systematic errors, which are on the order of 0.03 mag. Reddening corrections have not been applied, but the reddening vector in $E\left(g^{*}-r^{*}\right)$ (as determined from Schlegel, Finkbeiner, \& Davis 1998) is given in (11). The last column indicates the source catalog of each object. A " 1 " indicates that the object is in the NED catalog. SDSS quasars discovered during follow-up observations are represented by a " 2 ." Objects found in more than one catalog are so indicated.

Three quasars were both NED and SDSS sources, and had large discrepancies in their redshifts as reported by NED. The following quasars from NED have incorrect redshifts: UM 203, UM 183, and UM 427. Their correct redshifts as derived from SDSS spectroscopic commissioning data are $1.47,1.14$, and 1.69 , respectively. We further note that PC $0036+0032(z=4.51)$ is not included because it is improperly classified by NED as a galaxy. Since not all previously known quasars in this area of the sky have new SDSS spectra, it is likely that there are other known quasars in this region with incorrect redshifts or that are misclassified.

\section{QUASAR COLORS}

We present SDSS quasar color-color diagrams in Figure 4 , where we plot $\left(u^{*}-g^{*}, g^{*}-r^{*}\right),\left(g^{*}-r^{*}, r^{*}-i^{*}\right)$, and $\left(r^{*}-i^{*}, i^{*}-z^{*}\right)$, respectively. The black points and black contours are 10,000 stellar sources brighter than $i^{*}=19$ taken from the run 745 data [Galactic $(l, b)=\left(248^{\circ}, 48^{\circ}\right)$ to $\left.\left(18^{\circ}, 27^{\circ}\right)\right]$, which covers approximately the same region of space as run 756. The color points are the quasars, colorcoded according to their redshifts: blue points are lowredshift quasars, and red points are high-redshift quasars. Only quasars whose errors are small $(\leq 0.2 \mathrm{mag}$ each of the three relevant bands) are plotted. The solid black line is the median color-color track of the entire sample. The average Galactic reddening and an extragalactic (internal) reddening vector (defined in $\S 5.1$ ) are also plotted in the bottom right-hand corner of each panel in Figure 4. In each case, the uppermost vector is the average Galactic reddening vector. Throughout the paper all figures and tables (except Table 2) use magnitudes and colors that have been dereddened using the reddening maps of Schlegel et al. (1998).

The broadband SDSS colors of quasars as a function of redshift are presented in tabular form in Table 3 and in graphical form in Figure 5. Only quasars whose errors are less than $0.1 \mathrm{mag}(10 \sigma$ detections $)$ in each of the magnitudes that contribute to a given color are included. In each redshift bin, we measure the median colors of the quasars, as well as the limits within which $95 \%$ of the quasars lie, which we hereafter refer to as "confidence limits." Table 3 gives the median and $95 \%$ confidence limits of the dereddened colors of quasars in the redshift bins given, plus the number of quasars per bin. In Figure 5, the black dots are point sources as seen by SDSS, whereas magenta dots are extended sources (i.e., the profile is not suitably described by 

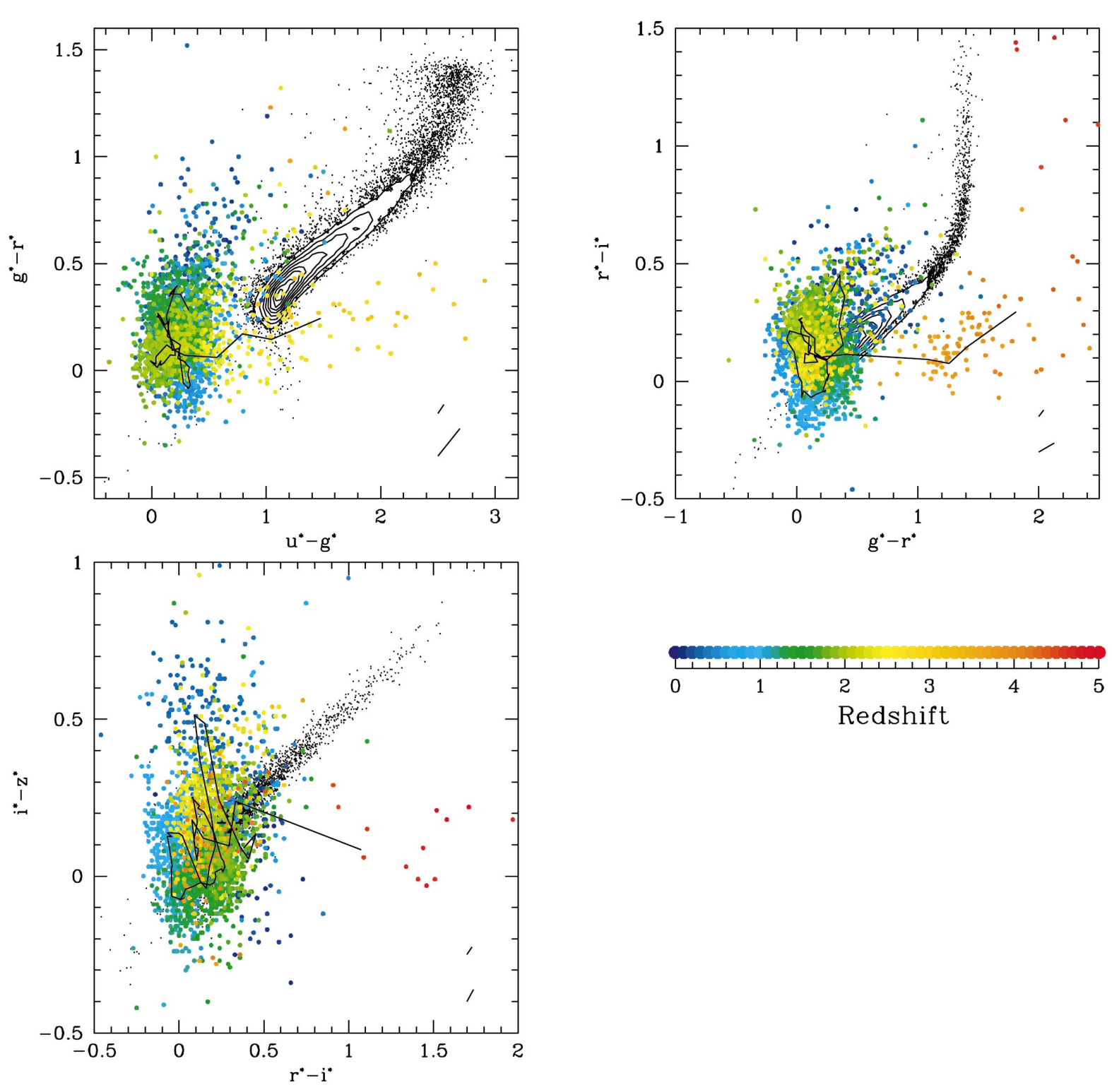

FIG. 4.-SDSS color-color diagrams for 2625 quasars (color points) and 10,000 stars (black points and black contours). Only objects with errors less than 0.2 mag in each band are shown. Quasar points are coded as a function of redshift, where the redshift is given by the color as indicated in the legend. The long solid black lines are the median color-color tracks of the quasars. Galactic (upper) and extragalactic (lower) reddening vectors are given in the bottom right-hand corner of each panel, where the Galactic reddening vector is the mean over the whole sample.

a point-spread function, as determined by the SDSS photometric pipeline) with $z \leq 0.6$. The solid light blue line is the median color-redshift vector for these quasars in redshift bins of size $\Delta z=0.05$ from $z=0.05$ to $z=2.1$, as reported in Table 3. Starting with $z=2.2$ the bin size is $\Delta z=0.2$; the $z=2.2$ bin partially overlaps the $z=2.1$ bin. The median color-redshift relation in Figure 5 is smoothed by $50 \%$ of the bin size for plotting purposes. We use the median to describe the color-redshift vector rather than a mean, since the median is less sensitive to outliers. The dashed red line is similar to the median color-redshift track from Fan (1999); see $\S 4.4$ for further discussion. Two vectors are plotted as solid lines in the bottom right-hand corner of each panel. The one on the left shows the magnitude of typical Galactic reddening, whereas the one on the right is representative of the change in color due to extragalactic reddening. These are the same vectors as in Figure 4. We discuss reddening in further detail in $\S 5.1$.
The high quality of the data is quite apparent in Figure 4 and can be compared with similar graphs using photometry from photographic plates (e.g., Warren et al. 1991b; Irwin et al. 1991). The tightness of the stellar locus is a result of the high-quality CCD photometry. Clearly, more accurate photometric data allow quasars to be found more easily. A lack of color degeneracy in the quasar colors as a function of redshift is apparent in the color-color (Fig. 4) and the color-redshift diagrams (Fig. 5). Quasars with similar redshifts tend to have the same colors, whereas quasars with different redshifts occupy different places in color space. The fact that there is so much structure in the color-redshift relation and that the scatter in the colors at a given redshift is reasonably small may allow for the determination of photometric redshifts for quasars (Richards et al. 2000). Photometric redshifts are now common practice for galaxies (e.g., Budavári et al. 2000) and have recently become possible for quasars (Wolf et al. 2000). 
TABLE 3

COLOR-REDShIFT RELATION

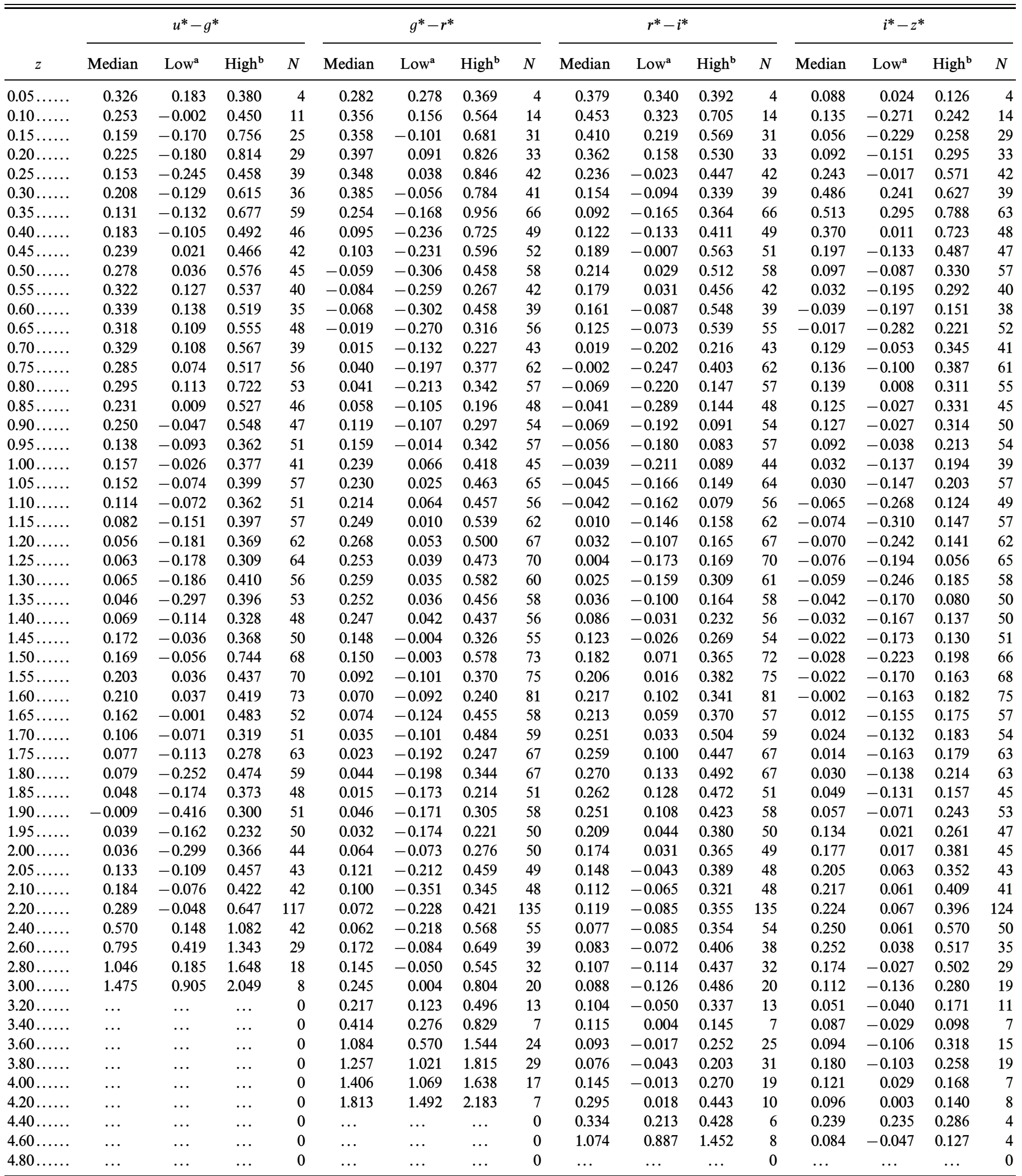

${ }^{\text {a }}$ Color at the $95 \%$ confidence limit to the blue of the median.

${ }^{\mathrm{b}}$ Color at the $95 \%$ confidence limit to the red of the median. 


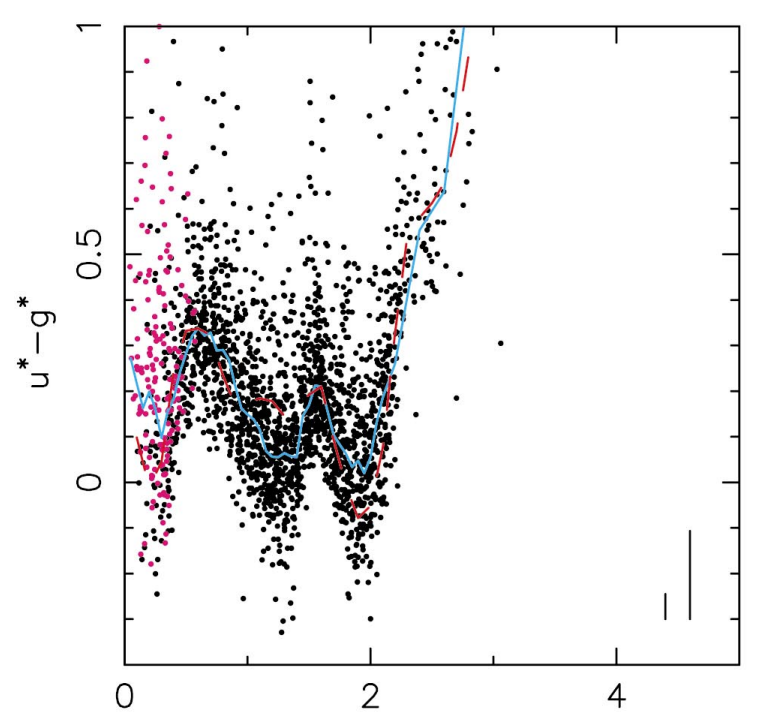

Z

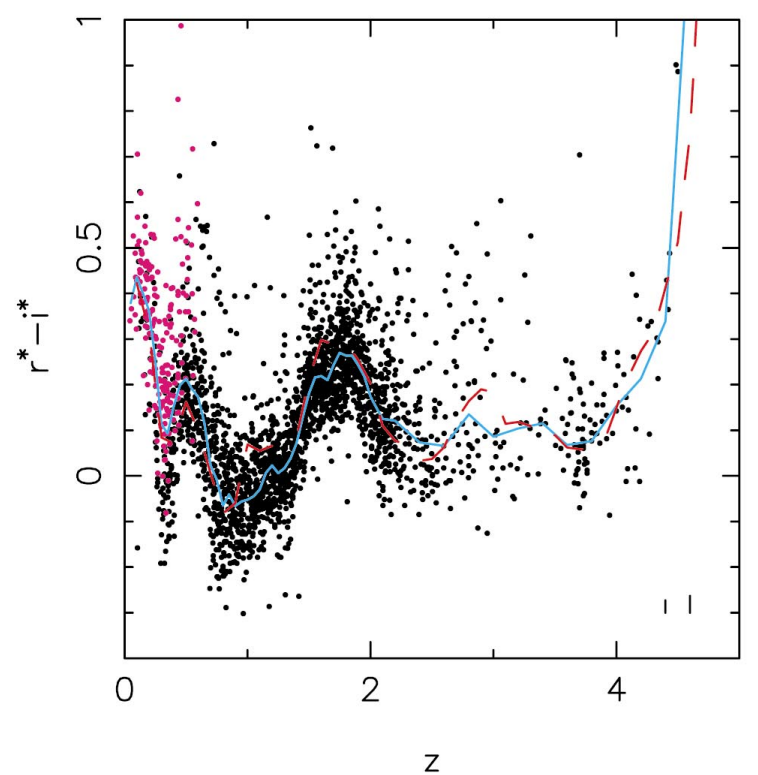

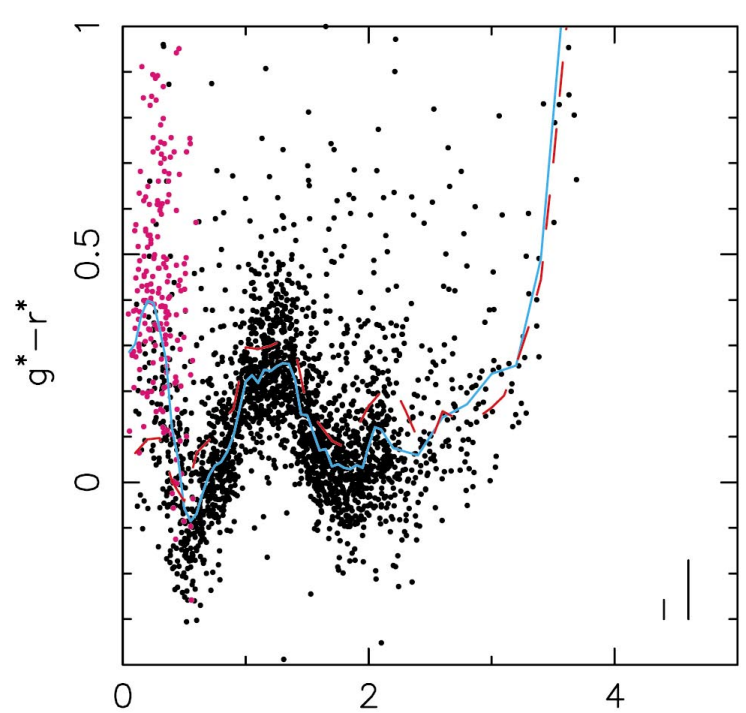

Z

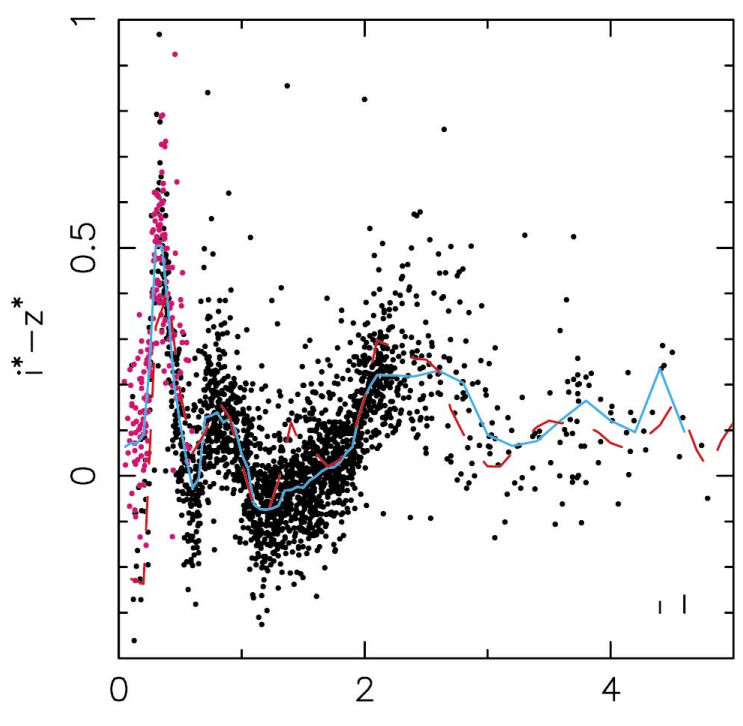

FIG. 5.-SDSS color vs. redshift relation for 2625 quasars (black dots: point sources; magenta dots: extended sources with $z \leq 0.6)$. Only objects with errors less than $0.1 \mathrm{mag}$ in all bands are shown. The median in redshift bins of $0.05(z \leq 2.2)$ and $0.2(z>2.2)$ is given by the solid light blue line (smoothed by $50 \%$ of the bin size). The dashed red line is a modified version of the simulated median quasar color-redshift relation from Fig. 12 of Fan (1999). Galactic (leftmost) and extragalactic (rightmost) reddening vectors are given in the bottom right-hand corner of each panel.

\subsection{Theoretical Colors}

We begin our analysis with a discussion of the expected colors of quasars in the SDSS photometric system. For a general introduction on the subject see Fan \& Chen (1994) and Warren et al. (1994), or Fan (1999) for a discussion specific to the SDSS. To first order, the spectra of quasars can be characterized as a power law. A power-law spectrum has the convenient property that it has the same color at any redshift. The color of an object with flux density $f(v)$ is given by

$$
\begin{aligned}
m_{1} & -m_{2}=-2.5 \\
\times & {\left[\log \frac{\int f(v) S_{1}(v) d \log v}{\int S_{1}(v) d \log v}-\log \frac{\int f(v) S_{2}(v) d \log v}{\int S_{2}(v) d \log v}\right], }
\end{aligned}
$$

where $S_{1}(v)$ and $S_{2}(v)$ are the throughput of the system in each bandpass (for a photon counting system). For the
SDSS photometric system, there is no additive constant since the SDSS magnitudes are on the $A B$ system (Oke \& Gunn 1983; Fukugita et al. 1996). This lack of an additive constant is in contrast with the $U B V R I$ system, which is Vega based; the computation of colors in the UBVRI system requires a correction for the fact that the spectrum of Vega is not perfectly flat in $f_{v}$. For a flat-spectrum source $\left(\alpha_{v}=0\right)$, the colors in an $A B$ system are always 0 . Table 4 gives the expected SDSS colors for a range of spectral indices. The spectral indices are given in both frequency and wavelength units, where $\alpha_{\lambda}$ and $\alpha_{v}$ are defined such that $\alpha_{\lambda}=-\left(2+\alpha_{v}\right), f_{v} \propto v^{\alpha_{v}}$ and $f_{\lambda} \propto \lambda^{\alpha_{\lambda}}$. Expected colors for other spectral indices can be interpolated or extrapolated from those given in Table 4.

From Figure 5 it is clear that the colors of quasars are not strictly power laws; however, the average colors are consistent with the input power-law distribution of Fan (1999), 
TABLE 4

POWER-LAW COLORS OF QUASARS

\begin{tabular}{crrrrr}
\hline \hline$\alpha_{v}$ & \multicolumn{1}{c}{$\alpha_{\lambda}$} & $u^{\prime}-g^{\prime}$ & $g^{\prime}-r^{\prime}$ & $r^{\prime}-i^{\prime}$ & $i^{\prime}-z^{\prime}$ \\
\hline$-2.5 \ldots \ldots$ & 0.5 & 0.756 & 0.739 & 0.523 & 0.459 \\
$-2.0 \ldots \ldots$ & 0.0 & 0.602 & 0.594 & 0.419 & 0.367 \\
$-1.5 \ldots \ldots$ & -0.5 & 0.450 & 0.447 & 0.315 & 0.275 \\
$-1.0 \ldots \ldots$ & -1.0 & 0.299 & 0.299 & 0.210 & 0.184 \\
$-0.5 \ldots \ldots$ & -1.5 & 0.149 & 0.150 & 0.105 & 0.092 \\
$0.0 \ldots \ldots \ldots$ & -2.0 & 0.000 & 0.000 & 0.000 & 0.000 \\
\hline
\end{tabular}

$\alpha_{v}=-0.5 \pm 0.3$. Whereas the power-law index of the quasar spectrum sets the average colors of a quasar, the colors will deviate from this value as a result of emission and absorption features (e.g., the Ly $\alpha$ forest). To identify the nature of these discrepancies, we turn to an analysis of the expected colors of quasars using a composite SDSS quasar spectrum convolved with the SDSS transmission curves.

\subsection{Composite Spectrum Colors}

A composite quasar spectrum has been constructed from the early SDSS spectroscopic commissioning spectra (Vanden Berk et al. 2000; Vanden Berk et al. 2001). The composite spectrum extends from 1050 to $7000 \AA \AA$ in the rest frame. We have convolved this spectrum with the most recent SDSS transmission curves (Fan et al. 2001, Appendix A). The resulting color-redshift tracks for the composite spectrum are presented in Figure 6.

In Figure 6 the solid blue line is the color-redshift track of the composite spectrum. For comparison, we also replot the empirical median color-redshift tracks from Figure 5 (solid black line) and the expected colors from a power-law spectrum of the form $f_{v} \propto v^{-0.5}$ (dashed black line; see Table 4).
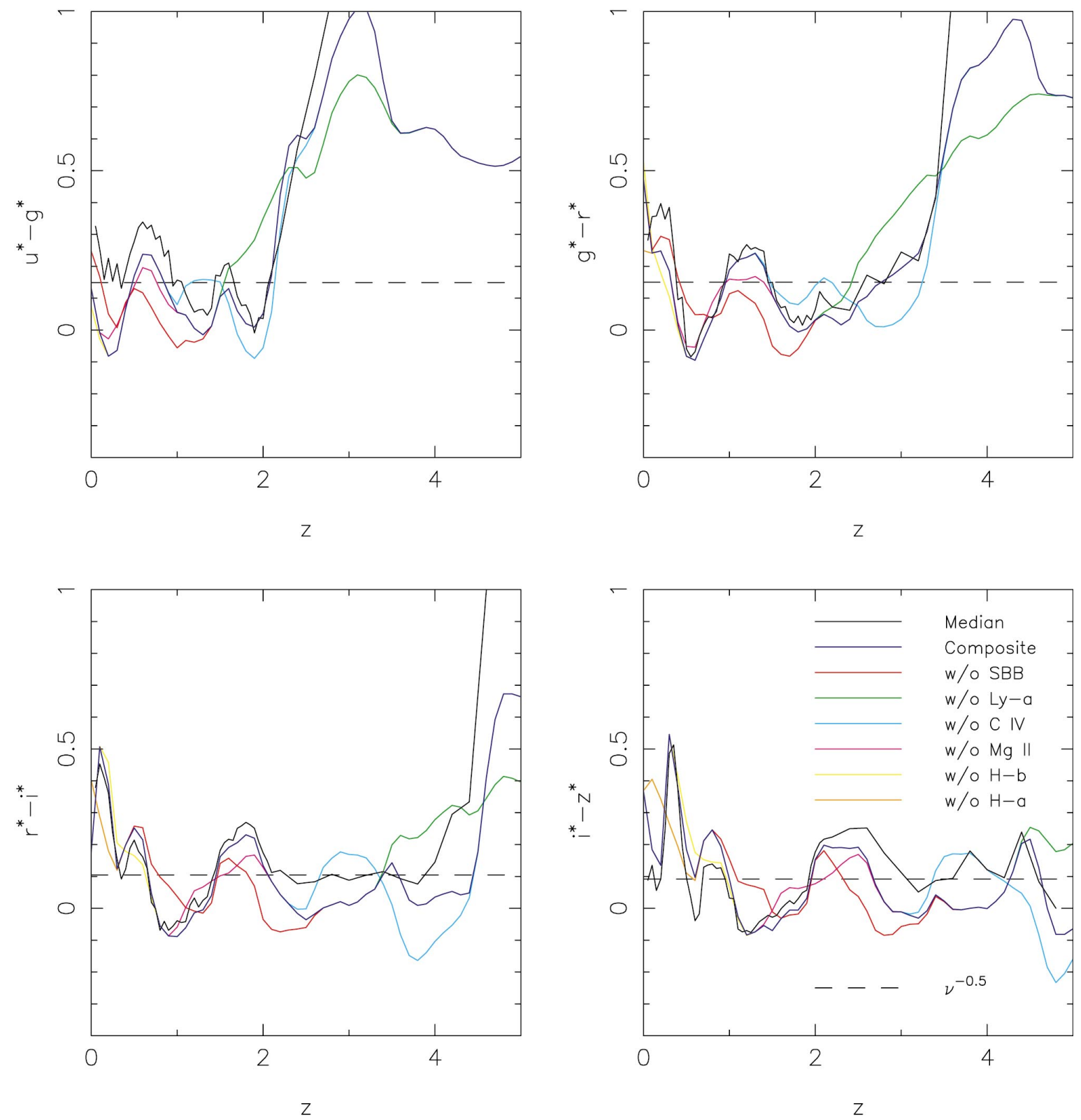

Fig. 6.-Simulated color vs. redshift. The solid black line is the observed median color-redshift relation from Table 3. The dashed black line is the color for a power-law spectrum of the form $f_{v} \propto v^{-0.5}$. The solid blue line is color-redshift track of the SDSS quasar composite spectrum (Vanden Berk et al. 2000, 2001). The remaining lines show the colors as a function of redshift when specific emission features are removed from the composite spectrum. 
The remaining lines in Figure 6 show what happens when a given emission feature is cut out of the composite spectrum and is replaced with a power law; i.e., each emission line is removed from the composite spectrum, and then the flux values in the wavelength range removed are replaced by a power-law spectrum of the form $f_{v} \propto v^{-0.3}$ normalized at $1450 \AA$, which is a good fit to the composite spectrum (Vanden Berk et al. 2000; Vanden Berk et al. 2001). A small additive constant was also required to make the spliced region fit into the spectrum cleanly. Since the strength of the Ly $\alpha$ forest is a function of redshift and because the composite does not cover the entire optical spectrum for $0<z<5$, the ends of the composite spectrum are padded with the average value between 1050 and $1150 \AA$ at the blue end, and 7000 and $8000 \AA$ at the red end. As a result, the absolute colors may not be accurate in the blue colors at high redshift and in the red colors at low redshift. Figure 6 displays what happens when $\mathrm{Ly} \alpha, \mathrm{C}$ IV, $\mathrm{Mg}$ II, $\mathrm{H} \beta, \mathrm{H} \alpha$, or the small blue ( $\lambda 3000)$ bump (SBB) are removed. In $\S 4.4$, we will demonstrate the effect that a strong emission line has on the colors of an otherwise power-law spectrum. A knowledge of this effect is helpful to understanding the results in the next section.

\subsection{Empirical Colors}

The color-redshift relations of quasars in the SDSS bandpasses exhibit considerable structure. We attempt to describe the causes of the features in the color-redshift diagrams. Features in the color-redshift relations are identified by comparing the results from the composite spectrum above (Fig. 6) with the measured colors of quasars (Fig. 5). All colors used for this analysis are dereddened colors. The emission lines that significantly affect the broadband colors include $\mathrm{Ly} \alpha, \mathrm{C}$ IV, $\mathrm{Mg}$ II, $\mathrm{H} \alpha, \mathrm{H} \beta$, and the $\lambda 3000$ bump. The $\lambda 3000$ bump consists of Balmer continuum emission and $\mathrm{Fe}$ II emission features (Grandi 1982; Peterson 1997). The feature starts at approximately $2300 \AA$ and extends to approximately $3800 \AA$. The $\mathrm{Mg}$ II emission line is nearly centered between these ranges and contributes significantly to color changes as a function of redshift since it sits on top of the $\lambda 3000$ bump.

We identify each of the significant features in the colorredshift curves. Many of the features in the color-redshift relation are caused by more than one feature in the quasar spectrum interacting with the transmission curves. A more detailed understanding of the causes of deviations from power-law colors can be obtained by comparing Figure 6 with Figure 5. Understanding the causes of the colorredshift features is interesting in and of itself, but also is helpful for determining sample completeness for objects with particularly weak or strong emission features.

$$
\text { 4.3.1. } u^{\prime}-g^{\prime}
$$

$z \sim 0.1$ to 0.4 . - The colors in this region of redshift have a considerable range; the range is much larger than for the redder SDSS colors. A comparison of the composite spectrum colors with the composite spectrum colors without the $\lambda 3000$ bump reveals that some of this range could be due to objects having a wide range of $\lambda 3000$ bump strengths, but see $\S 5.1$ for another explanation.

$z \sim 0.3$. - The $\lambda 3000$ bump and $\mathrm{Mg}$ II are in $u^{\prime}$ and cause the color to be blue.

$z \sim 0.6$. - The $\lambda 3000$ bump and $\mathrm{Mg}$ II are in $g^{\prime}$, making the color red. $z \sim 1.3$. - $\mathrm{C}$ IV is in $u^{\prime}$ and shifts the color to the blue.

$z \sim 1$.6.- $\mathrm{Ly} \alpha$ is entering $u^{\prime}$, while $\mathrm{C}$ IV is leaving $u^{\prime}$ and entering $g^{\prime}$.

$z \sim 1.9$. - $\mathrm{Ly} \alpha$ is in $u^{\prime}$, but the effect is tempered by the presence of C IV in $g^{\prime}$. The strength of Ly $\alpha$ dominates nevertheless, causing a blue dip.

$z \sim 2.3$ to 2.4.-A small plateau-like feature is caused as C IV leaves $g^{\prime}$ shortly after Ly $\alpha$ enters $g^{\prime}$.

$z>2.6$. - The $u^{\prime}-g^{\prime}$ color rises rapidly as the Ly $\alpha$ forest and Lyman-limit systems cause there to be little or no flux in the $u^{\prime}$ band.

$$
\text { 4.3.2. } g^{\prime}-r^{\prime}
$$

$z \sim 0.2$ to 0.3 . - The presence of $\mathrm{H} \beta$ in $r^{\prime}$ keeps the average color relatively red.

$z \sim 0.5$. - The $\lambda 3000$ bump is mostly in $g^{\prime}$, causing the color to be blue. As with the $z<0.4$ region in $u^{\prime}-g^{\prime}$, there is evidence for a population of redder objects.

$z \sim 1.2$. - The combination of $\mathrm{Mg}$ II and the $\lambda 3000$ bump in $r^{\prime}$ cause a reddening near this redshift.

$z \sim 1.75$. - The $\lambda 3000$ bump and C IV offset each other as the latter enters $g^{\prime}$ and the former leaves $r^{\prime}$.

$z \sim 2.1$. - The presence of $\mathrm{C}$ IV in $g^{\prime}$ keeps the color bluer than a power law.

$z \sim 2.5$ to 3.5.- The $\mathrm{C}$ IV line pushes the color to the red, whereas Ly $\alpha$ pushes the color to the blue. As the redshift increases more and more of the Ly $\alpha$ forest enters $g^{\prime}$, and the color reddens.

$z>4.0$. - The $g^{\prime}-r^{\prime}$ color rises rapidly as the Ly $\alpha$ forest and Lyman-limit systems cause there to be little or no flux in the $g^{\prime}$ band.

$$
\text { 4.3.3. } r^{\prime}-i^{\prime}
$$

$z \sim 0.1$ to 0.2 . $-\mathrm{H} \alpha$ is in $i^{\prime}$ and makes the color redder than the average power-law value.

$z \sim 0.3$. - The presence of $\mathrm{H} \beta$ in $r^{\prime}$ drives the color blueward, which is enhanced when $\mathrm{H} \alpha$ leaves $r^{\prime}$.

$z \sim 0.5$. - The color moves back to the red while $\mathrm{H} \beta$ is in $i^{\prime}$.

$z \sim 0.9$. - The $\lambda 3000$ bump fills the $r^{\prime}$ filter making the color bluer.

$z \sim 1.2$. - A small kink in the median colors is noticeable here as the $\lambda 3000$ bump and $\mathrm{Mg}$ II trade off in influence in the $r^{\prime}$ filter.

$z \sim 1.4$ to 1.5 .- The color makes a sharp transition from blue to red as $\mathrm{Mg}$ II leaves $r^{\prime}$ and enters $i^{\prime}$.

$z \sim 1.65$. - A small kink in the median colors is noticeable here as the $\lambda 3000$ bump and $\mathrm{Mg}$ II trade off in influence in the $i^{\prime}$ filter.

$z \sim 1.8$. - A small hump is caused as both $\mathrm{Mg}$ II and the $\lambda 3000$ bump push the color redward.

$z \sim 2.0$ to 2.5.-The color is driven back to the blue as the $\lambda 3000$ bump leaves $i^{\prime}$.

$z \sim 2.6$ to 3.4. - C IV keeps the color blue, when it would otherwise have reddened.

$z \sim 3.4$ to 4.4.- - Ly $\alpha$ and C IV offset each other during the period when the former is in $r^{\prime}$ and the latter is in $i^{\prime}$.

$z>4.5$. - The $r^{\prime}-i^{\prime}$ color rises rapidly as the Ly $\alpha$ forest and Lyman-limit systems cause there to be little or no flux in the $r^{\prime}$ band.

$$
\text { 4.3.4. } i^{\prime}-z^{\prime}
$$

$z \sim 0.2$. $-\mathrm{H} \alpha$ is in $i^{\prime}$ and keeps the color blue.

$z \sim 0.3$ to 0.4 . $-\mathrm{H} \alpha$ has moved into $z^{\prime}$, causing an abrupt reddening. 
$z \sim 0.6$. $-\mathrm{H} \beta$ is in $i^{\prime}$, whereas $\mathrm{H} \alpha$ is leaving $z^{\prime}$, resulting in a blue color.

$z \sim 0.8$. $-\mathrm{H} \beta$ has moved into $z^{\prime}$ and causes the color to become redder.

$z \sim 1.2$. - The $\lambda 3000$ bump in $i^{\prime}$ drives the color blueward.

$z \sim 1.9$ to 2.1 . $-\mathrm{Mg}$ II moves from $i^{\prime}$ to $z^{\prime}$, causing an abrupt reddening.

$z \sim 2.1$ to 2.5 .-As with the $z \sim 1.7$ feature in $r^{\prime}-i^{\prime}$, a small red hump is formed as both $\mathrm{Mg}$ II and the $\lambda 3000$ bump push the color to the red.

$z \sim 3.4$ to 4.2 . - C IV keeps the color bluer than the average power-law color.

$z \sim 4.4$ to 5.0.- - Ly $\alpha$ and $\mathrm{C}$ IV offset each other as C IV pushes the color redward at the same time that Ly $\alpha$ pushes the color blueward.

\subsection{Comparison of Expected and Measured Colors}

To characterize the differences between the expected colors of a power law and the empirical colors of quasars, it is instructive to know what effect the profile of a strong emission line has on the colors of quasars as the line is redshifted through each of the filters. Figure 7 shows what happens when a top-hat emission line with an observed equivalent width of $200 \AA$ that is $20 \AA$ wide is redshifted through each of the filters. The emission line starts centered at $3000 \AA(z=0)$ where the $u^{\prime}$ filter begins and ends at 11500 $\AA(z=2.833)$ where the $z^{\prime}$ filter ends. The four curves are $u^{\prime}-g^{\prime}, g^{\prime}-r^{\prime}, r^{\prime}-i^{\prime}$, and $i^{\prime}-z^{\prime}$, respectively, with $u^{\prime}-g^{\prime}$ being the curve in the lower left. These features are similar to the effect that the Ly $\alpha$ emission line has on the broadband colors of quasars. Note the similarity of the dip in $u^{*}-g^{*}$ near $z=1.9$ in Figure 5 to the dip near $z=0.2$ in Figure 7 .

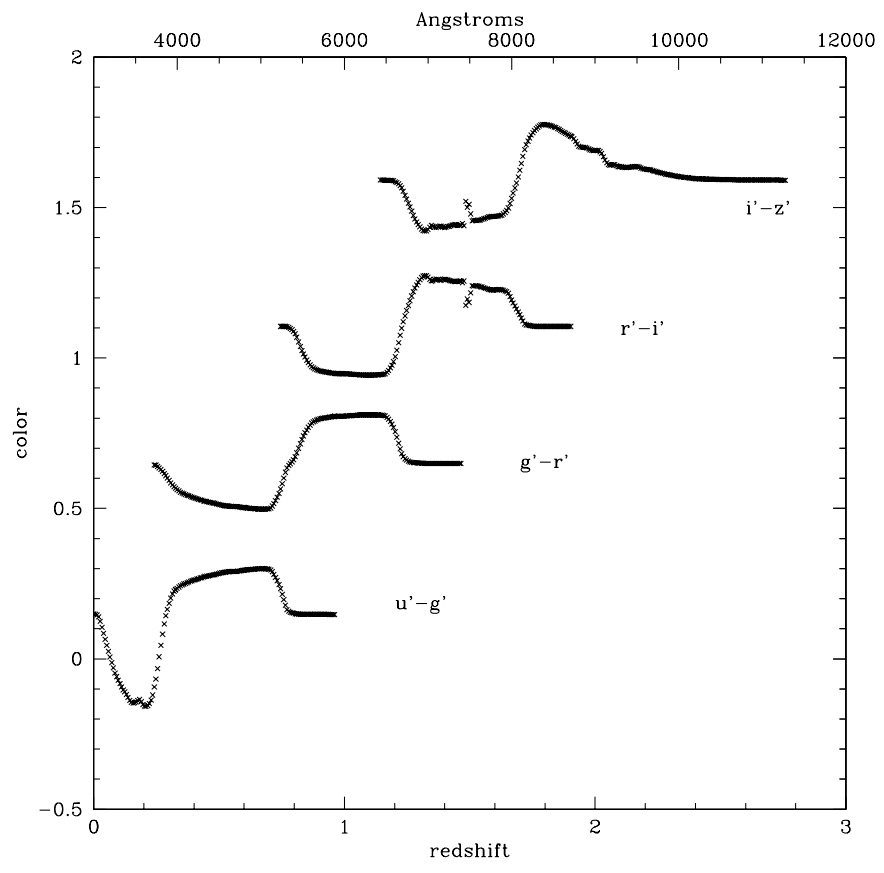

FIG. 7.-Color vs. redshift for a top-hat emission line superposed upon a power-law continuum $\left(\alpha_{v}=-0.5\right)$. The line center of the top hat begins at $3000 \AA$ and is redshifted through each of the transmission curves. The lower axis gives the redshift of the line, whereas the upper axis gives the wavelength of the line. The top hat has an observed equivalent width of $200 \AA$ and is $20 \AA$ wide. The bottom, left-hand curve is the $u^{\prime}-g^{\prime}$ curve. The other colors are shifted in the $y$-direction by 0.5 each.
A broad feature, such as is characterized by $\mathrm{Fe}$ II emission, has a similar profile, although the slope of the color change between bandpasses is less striking. It is particularly interesting that a relatively weak, but broad, emission features such as the $\mathrm{Fe}$ II complexes can have an equal effect to that of strong, narrow emission feature such as Ly $\alpha$. The total equivalent width within the bandpass matters much more than the manner in which that equivalent width is distributed, since the bandpasses are broad. For example, note the similarity of the $r^{*}-i^{*}$ colors from $z=0.6$ to $z=2.4$ and the $i^{*}-z^{*}$ colors from $z=0.8$ to $z=3.0$ in Figure 5 to the $r^{\prime}-i^{\prime}$ and $i^{\prime}-z^{\prime}$ shapes, respectively, in Figure 7. These features are produced by a combination of the $\lambda 3000$ bump and $\mathrm{Mg}$ II emission.

One of the most powerful uses of this analysis is the refinement of the simulated quasar spectrum, which, in turn, tells us about the empirical properties (spectral indices, emission line equivalent widths, etc.) of quasars. Fan (1999) calculated theoretical colors of quasars in the SDSS filter system as a function of redshift. Although these simulated colors are correct to first order (they correctly reproduce the colors of quasars on the ensemble average), there are deviations from the average that have a significant impact upon the colors of quasars as a function of redshift. The simulated quasar color-redshift track shown in Figure 5 is similar to that of Fan (1999), but uses a larger equivalent width for the $\mathrm{Fe}$ II features and the updated filter curves. This theoretical color-redshift relation matches the observed color-redshift relation surprisingly well. While the simulation does indeed fit the data quite well, there are still regions of color-redshift space where the simulations and the data do not agree well.

\subsubsection{The $\lambda 3000$ Bump}

One piece of information that we can glean from a comparison of the simulated color-redshift tracks with the observed tracks is the structure of the $\lambda 3000$ bump. The quality of the digital photometry provided by the SDSS allows us to determine the structure of this spectral feature without having to examine any spectra. Such an analysis is of particular interest given the role that the $\lambda 3000$ bump plays in the spectra of quasars (Netzer et al. 1985).

Some of the most notable deviations of the empirical colors from the simulated colors occur at $z=1.0$ and $z=1.4$ in $r^{*}-i^{*}$ and $i^{*}-z^{*}$, respectively. The sharp break in the simulated colors from Figure 5 is caused by the fact that the $\mathrm{Fe}$ II emission surrounding the $\mathrm{Mg}$ II emission line has been modeled as two distinct features following Francis et al. (1991). However, a comparison with the empirical color-redshift track shows that the two bumps on either side of $\mathrm{Mg}$ II actually combine to form an apparent continuum, such that $\mathrm{Mg}$ II lies well above the power-law continuum level. As a result, the regions surrounding $\mathrm{Mg}$ II emission are not representative of the power-law continuum spectrum. In addition to the merging of what Francis et al. (1991) call " $\mathrm{Fe}$ II feature 2 " and " $\mathrm{Fe}$ II feature 3 ", it is also quite possible that "Fe II feature 1" (longward of C IV emission) merges with both of these and that the dip near $2200 \AA$ in the flux of composite quasar spectra is caused by dust (Richstone \& Schmidt 1980; but see Oke et al. 1984 for a counter-argument).

The effect of the $\lambda 3000$ bump at $z \leq 0.6$ is also quite evident. For $r^{*}-i^{*}$ and $i^{*}-z^{*}$ the colors of quasars are very tight at $z \leq 0.6$. There is considerable variation as a function of redshift, but very little scatter at a given redshift. 
This is not the case for $u^{*}-g^{*}$ and $g^{*}-r^{*}$, where there is a large range of colors. A possible explanation is that quasars can exhibit a broad range of strength in the $\lambda 3000$ bump at low redshifts. A closer examination of the definition of a quasar at low redshift is warranted (see $\S 5.5$ for more details and another possible explanation).

\subsubsection{IV}

There are significant differences between the simulated and measured colors of quasars in the redshift ranges affected by $\mathrm{C}$ IV. Note in particular the $z \sim 1.3$ region in the $u^{*}-g^{*}$ panel and the $z \sim 2.1$ region in the $g^{*}-r^{*}$ panel of Figures 5 and 6 . A possible explanation for these discrepancies is that the assumed equivalent width for $\mathrm{C}$ IV is considerably smaller than the average equivalent width of $\mathrm{C} \mathrm{IV}$ in our sample. This is surprising because the $\mathrm{C}$ IV equivalent width in the simulation is taken from the LBQS composite quasar spectrum (Francis et al. 1991), and LBQS quasars contribute significantly to our sample. However, as with $\mathrm{Mg}$ II and the $\lambda 3000$ bump, C IV may be influenced by other emission, in particular lines of $\mathrm{Fe}$ II (1700 to $2200 \AA$ ), He II $\lambda 1640$, and $\mathrm{O}$ III] $\lambda 1663$. To the extent that these lines are missing from the simulations, the simulations will deviate from the empirical colors.

\subsection{3. $\mathrm{H} \alpha$}

A particularly useful diagnostic can be made from the effect of $\mathrm{H} \alpha$ on the broadband colors of SDSS quasars. That this is the case can be seen at $z \sim 0.2$ in the $i^{*}-z^{*}$ of Figure 5. The deviation of the simulated colors of Fan (1999) from the observed colors is caused by the fact that the simulations used the original transmission curves. It is now known that the true transmission curves deviate somewhat from those originally reported (Fan et al. 2001). The sharpness of the color-redshift feature in $i^{*}-z^{*}$ due to $\mathrm{H} \alpha$ can be used as a diagnostic throughout the course of the survey. Small changes in the transmission curves can be monitored as a function of time and as a function of CCD chip in the camera.

\section{DISCUSSION}

The primary purpose of this investigation of the empirical colors of quasars in the SDSS photometric system is to aid in the selection of quasars for spectroscopic observations during the course of the Sloan Digital Sky Survey. There are a number of complex issues involved in the selection of quasars in the most complete and efficient manner. The data presented herein provides a wealth of information that can be used to address a number of topics. We now turn to a discussion of some of the more important issues.

\subsection{Reddening}

Although the photometry presented herein has been corrected for Galactic reddening using the reddening maps of Schlegel et al. (1998), it is important to understand how much effect Galactic reddening has on the colors of quasars. The average Galactic reddening vector for the quasars in our sample is $(0.055,0.042,0.027,0.024)$ in $\left(u^{*}-g^{*}, g^{*}-r^{*}\right.$, $\left.r^{*}-i^{*}, i^{*}-z^{*}\right)$ coordinates. These vectors are plotted to scale as the leftmost of the two vectors in the lower righthand corner of the color-redshift curves in Figure 5 and the uppermost of the two vectors in the lower right-hand corner of the color-color plots in Figure 4. At low Galactic latitudes, Galactic reddening can be considerably more significant (see Fan 1999, Fig. 5).
In contrast to Galactic reddening, little is known about intergalactic reddening and reddening internal to quasars. Although it has been suggested that a significant fraction of quasars may be reddened and therefore missed in fluxlimited surveys (e.g., Fall \& Pei 1993; Francis et al. 1999), it is difficult to know exactly how prevalent internal reddening is and to what extent it effects the broadband colors of quasars. Close examination of the color-redshift relations in Figure 5 reveals that the spread in colors around the median color as a function of redshift is much larger for $u^{*}-g^{*}$ than for the other colors. Moreover, the scatter is asymmetric, in the sense expected for reddening.

To quantify this effect, we subtract the median colors given in Table 3 at each redshift from the colors of the quasars shown in Figure 5 (i.e., those with small photometric errors) with $0.4<z<3.0$ and examine the residual colors. Quasars that are classified as extended (as opposed to point sources) are excluded to avoid contamination from the host galaxy. Low-redshift objects are excluded to avoid confusion with Seyfert galaxies. High-redshift objects are excluded because of a relative paucity of data. Histograms of the residual colors are given in Figure 8. Note the red tail in the distribution of $u^{*}-g^{*}$ and, to a lesser extent, $g^{*}-r^{*}$. In order to determine the cause of this tail, we have created additional diagnostic plots.

The residual colors are plotted as a function of magnitude in the four panels of Figure 9, as a function of redshift in the four panels of Figure 10 and as a function of absolute magnitude $\left(q_{0}=0.5, H_{0}=65 \mathrm{~km} \mathrm{~s}^{-1} \mathrm{Mpc}^{-1}\right)$ in Figure 11. Except for Figures 10 and 11, Figures 8 to 12 exclude all quasars with $z<0.4$. Two sigma error bars for objects with magnitude 20 (in the bandpass given) are plotted in Figure 9 . Note that the scatter blueward of the median colors $(\equiv 0)$ for each pair of filters is less than the amount of scatter redward of the median colors. This scatter increases toward the red with fainter magnitudes, is more pronounced in the bluer colors, and is independent of redshift.

Such an effect is quite likely the result of reddening of the quasars, either external or internal, but certainly extragalactic; the fact that the reddening is independent of redshift points toward internal reddening over external reddening, since external reddening would depend on the volume of space enclosed at a given redshift. Table 5 gives the blue and the red one-sided $95 \%$ confidence deviations in each of the median-corrected colors for those objects brighter than $i^{*}=21$ and whose errors in each band are less than $0.1 \mathrm{mag}$. Also given are the corresponding values of spectral index.

We further demonstrate the reddening of some objects in Figure 12 where we plot the corrected colors against each other; typical errors are given in the top right-hand corner of each panel. The vector in each of the panels is the absolute value of the blue $95 \%$ confidence limit from Table 5. Objects whose red color excess is comparable to or larger

TABLE 5

SCATtER IN CORRECTED Colors (95\% CONFIDENCE)

\begin{tabular}{ccccc}
\hline \hline Color & $\Delta$ Blue Color & $\Delta$ Red Color & $\Delta \alpha$ Blue & $\Delta \alpha$ Red \\
\hline$u^{*}-g^{*} \ldots \ldots$ & -0.209 & 0.402 & 0.69 & 1.34 \\
$g^{*}-r^{*} \ldots \ldots$ & -0.190 & 0.319 & 0.64 & 1.07 \\
$r^{*}-i^{*} \ldots \ldots$ & -0.161 & 0.198 & 0.77 & 0.94 \\
$i^{*}-z^{*} \ldots \ldots$ & -0.173 & 0.211 & 0.95 & 1.15 \\
\hline
\end{tabular}



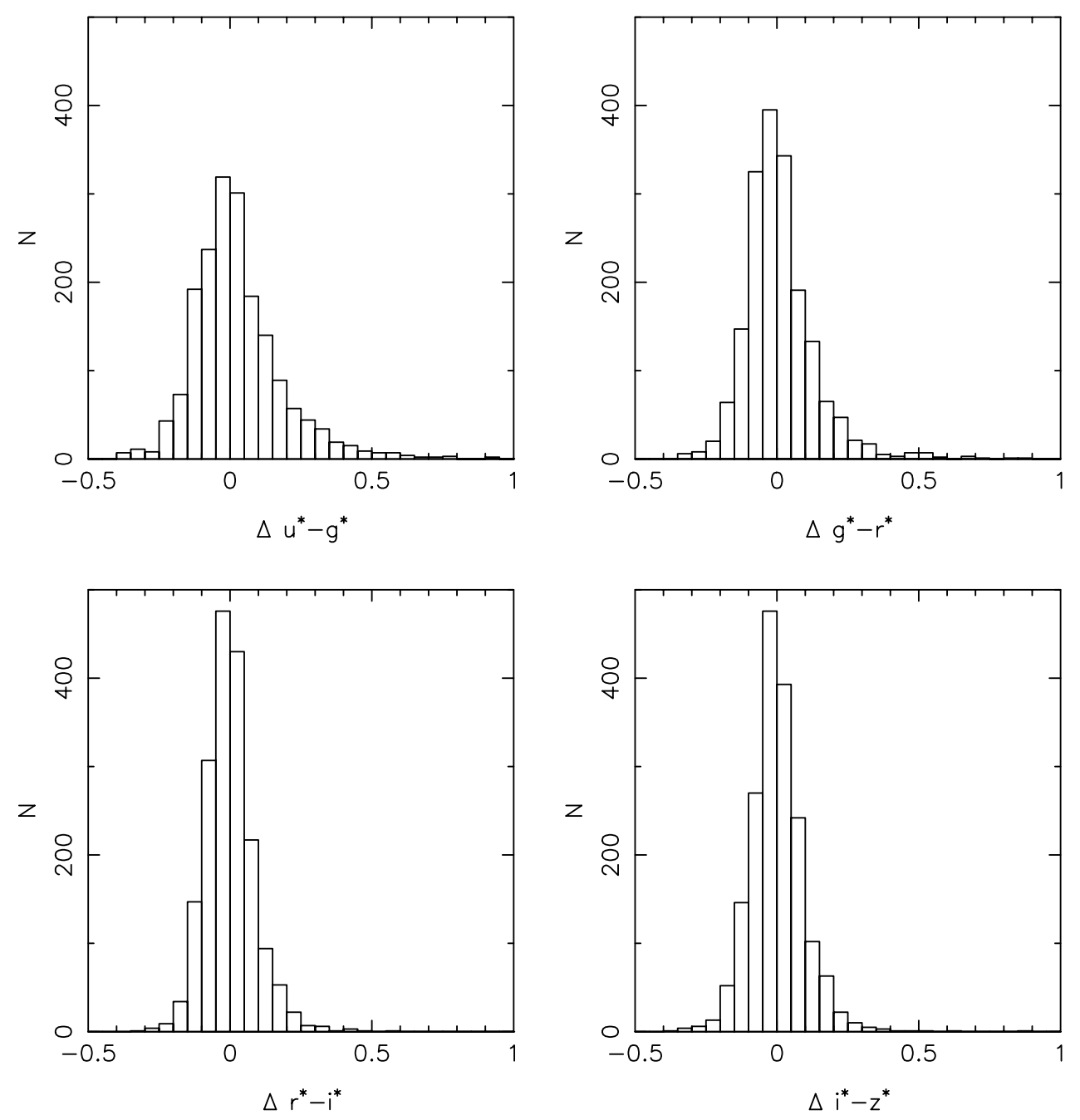

FIG. 8.-Histograms of observed quasar colors corrected by the median colors as a function of redshift

than the absolute value of the blue $95 \%$ confidence limit given in Table 5 show signs of reddening beyond what is expected from Galactic dust. The difference between the absolute value of the blue $95 \%$ confidence limit given in Table 5 and the red $95 \%$ confidence limit given in Table 5 yields an estimate of the amount of extragalactic reddening. The resulting vector $(0.193,0.129,0.037,0.038)$ is the extragalactic reddening vector used in Figures 4 and 5. This vector is similar in direction to the Galactic reddening vector.

We have defined a sample of 26 "reddened" quasars, which are those quasars whose red color excess in $u^{*}-g^{*}$, $g^{*}-r^{*}$, and $r^{*}-i^{*}$ lies outside the blue $95 \%$ confidence interval. Nearly half of these are radio sources, as we discuss below in $\S 5.3$. Some examples of these "red" quasars are given in Figure 13, which presents 10 of the 20 red quasars for which we have SDSS spectra. We show these spectra in order to demonstrate that these objects are truly redder than the average and are not simply the result of photometric errors, etc. Note that these spectra contain some broad absorption line (BAL) quasars, and many have strong, narrow absorption lines. These spectra can be compared with those presented in Figure 1, which shows the spectra of 10 more normal SDSS quasars spanning a range in redshift from $z \sim 0$ to $z \sim 5$.
The extent of the reddening in these quasars is a function of the assumed reddening curve. Whether the reddening is internal or external, features such as the $\lambda 2200$ bump (Mathis 1994), which is typically associated with reddening from dust grains, may influence the colors in a nonlinear manner. If the reddening is internal, it may come from depletion by material associated with the torus of gas and dust that is thought to surround the central quasar engine; if this is the case, reddening might be used as an orientation indicator for quasars that are not radio detected. Reddening might also arise in the quasar host galaxy or in other galaxies along the line of sight.

Reddened quasars can have colors that can be well removed from the predicted location of quasars in colorcolor space. Color-selected surveys may select against such quasars. Note in particular the dearth of faint red quasars in the tope right-hand panel of Figure 9. If there is a significant population of reddened quasars, they may constitute the optical counterparts of the remainder of the hard X-ray background that have hitherto gone undetected (Brandt et al. 2000; Mushotzky et al. 2000).

\subsection{Spectral Index Distribution}

Table 5 gives the blue and red one-sided range in spectral index ( $95 \%$ confidence) needed to produce the observed 

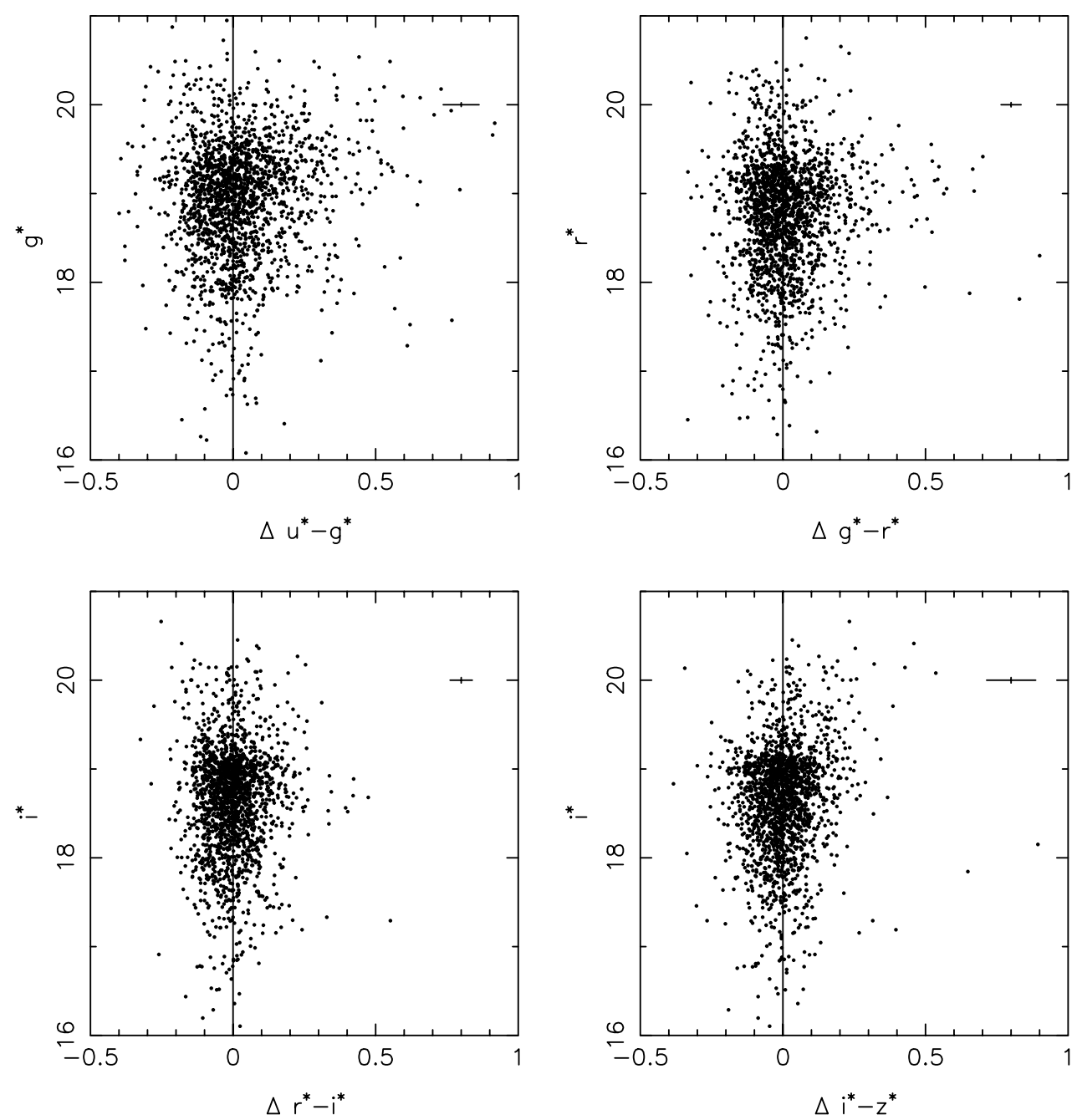

FIG. 9.- Observed quasar colors corrected by the median colors as a function of redshift, plotted as a function of magnitude. Two-sigma error bars in color and magnitude are given for the average $20^{\text {th }}$ magnitude object.

range of colors. If the blueward scatter in the colors is representative of the true (unreddened) scatter in optical spectral index, the spectral index distribution of the sample is approximately $\alpha_{v}= \pm 0.65$ (95\% confidence), where the error gives the range of values that includes $95 \%$ of sources with colors bluer than zero. Note that we have not determined the average spectral index from the data, but instead use $\alpha_{v}=-0.5$, which is typically used for the optical spectral indices of quasars and is not a bad fit to the data (Vanden Berk et al. 2001). A determination of the exact value of the average spectral index would require a large region of quasar spectra to be devoid of emission lines, which is not the case in the UV/optical part of the spectrum.

Another conclusion that may be drawn from Figure 10 is that the scatter in the colors of quasars at a given redshift must be primarily due to the range of optical spectral indices of the individual quasars. If the scatter in the colors was instead dominated by the strength of emission lines, then we would expect that this scatter would be a stronger function of redshift. In addition, as noted by Warren et al. (1994), a large intrinsic range of quasar spectral indices will blur the calculations of the luminosity function. Extrapolation of a magnitude to a different band using the wrong spectral index will give the wrong magnitude in the new band. For the observed range of spectral indices, the range of absolute $g^{*}$ magnitudes at $z=2$ given a selection function based on observed $i^{*}$ magnitudes is $\Delta M_{g^{\prime}}=0.89$.

\subsection{Radio Sources}

Although the vast majority of the quasars that the SDSS finds will be color-selected quasars, many will be radioselected. All FIRST sources with pointlike optical counterparts and $i^{*}<19$ will be targeted as quasar candidates. Targeting these radio-detected sources will assist in the determination of the completeness of our color-selected sample; using these objects, we will be able to test whether a significant fraction of quasars is being missed. At the present time, however, our sample of radio-detected quasars is not large enough to determine if the color-redshift distribution of these quasars are significantly different from that of color-selected objects.

We can, however, comment on the ensemble average of the colors of these quasars. For radio sources only, the blue scatter in the median corrected colors is consistent with scatter of \pm 0.64 in the spectral index ( $95 \%$ confidence). The scatter of the colors to the blue is not significantly different from the distribution of the sample as a whole. On the other hand, the $95 \%$ confidence limit in the red scatter requires 

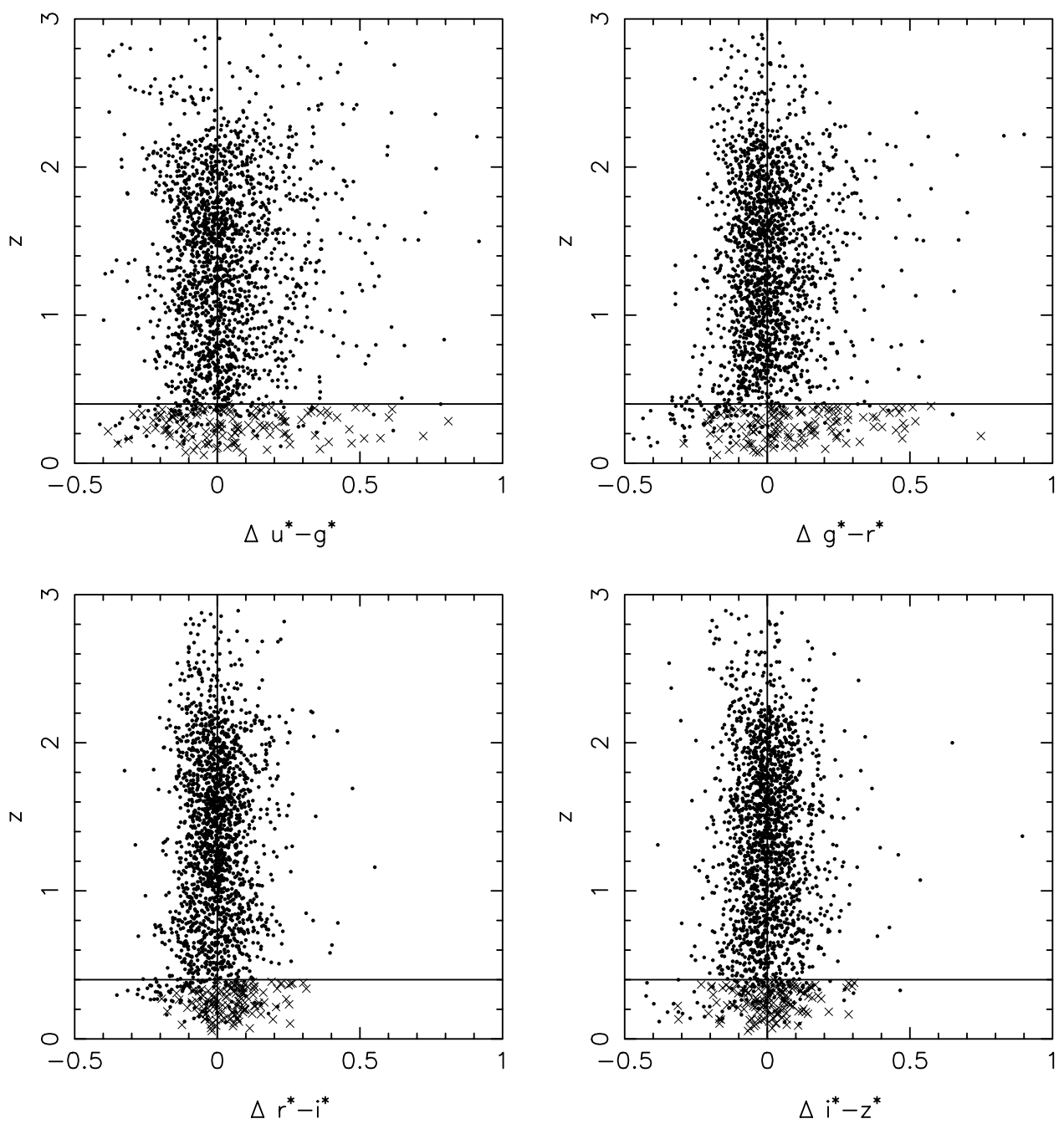

FIG. 10.-Quasar colors (corrected by the median colors as a function of redshift), plotted vs. redshift. Objects with $z \leq 0.4$ (horizontal line) are not used in the reddening analysis. Crosses mark extended sources with $z \leq 0.4$.

spectral indices redder than $\alpha_{v}=-1.5$, which is considerably redder than the sample as a whole. This result is consistent with the fact that many (12 of 26) of the reddened quasars discussed above are FIRST radio sources.

\subsection{UVX Color Selection}

Since the colors of $z<2.2$ quasars are dominated by the continuum power-law spectrum, it is expected that the UVX selection technique for these quasars should be relatively complete, i.e., their median colors are generally significantly bluer than the $u^{\prime}-g^{\prime}$ selection criterion. However, from Figure 5 we see that the $u^{*}-g^{*}$ color of $z \leq 2.2$ quasars can, in fact, be as red as or redder than $u^{*}-g^{*} \sim 0.6$. Quasars with $z \sim 0.1,0.6,1.6$, and 2.2 can have colors close to the UVX cutoff. As such, it is not possible to assume that the UVX technique is complete. Fluxlimited samples will indeed be incomplete near the flux limit as a result of larger scatter in the colors at the faint end of the distribution, and this incompleteness is a function of redshift. For example, Hawkins \& Veron (1993) have suggested that the fall-off in the number density of faint quasars as found by UVX quasar surveys such as Boyle et al. (1988) could be a result of this effect. One way to test this is to conduct a survey of faint quasar candidates to a limiting $g^{\prime}$ magnitude of $\sim 22$ (comparable to previous surveys) and compare the luminosity function of quasars in redshift regimes where median $u^{\prime}-g^{\prime}$ colors are much bluer than the UVX cutoff with those quasars in redshift regimes where median $u^{\prime}-g^{\prime}$ colors are very close to the UVX cutoff. Such a project would have to be conducted as a follow-up study to the SDSS using the data from the SDSS equatorial region in the south Galactic cap which will be imaged multiple times.

Additional problems with UVX quasar selection arise as a result of the colors of stars. At very bright magnitudes $\left(g^{*} \sim 16\right)$ there is very little stellar contamination in the UVX regime. However, at fainter limits, there is significant contamination of UVX quasars by stars, due to the metalpoor halo population. Figure 14 plots a color-magnitude diagram of blue stellar sources (black contours and black points) from run 756 , camera column 3 . At the bright end, the majority of the blue objects will be quasars, with some contamination from white dwarfs. As $g^{*}$ gets fainter, there are more and more stars that have blue colors. This is partly due to increasing photometric error at fainter magnitudes, but it is also likely to be the result of shifts in the stellar locus as a function of magnitude (and therefore metallicity) as discussed by Newberg et al. (1999) and Finlator et al. 

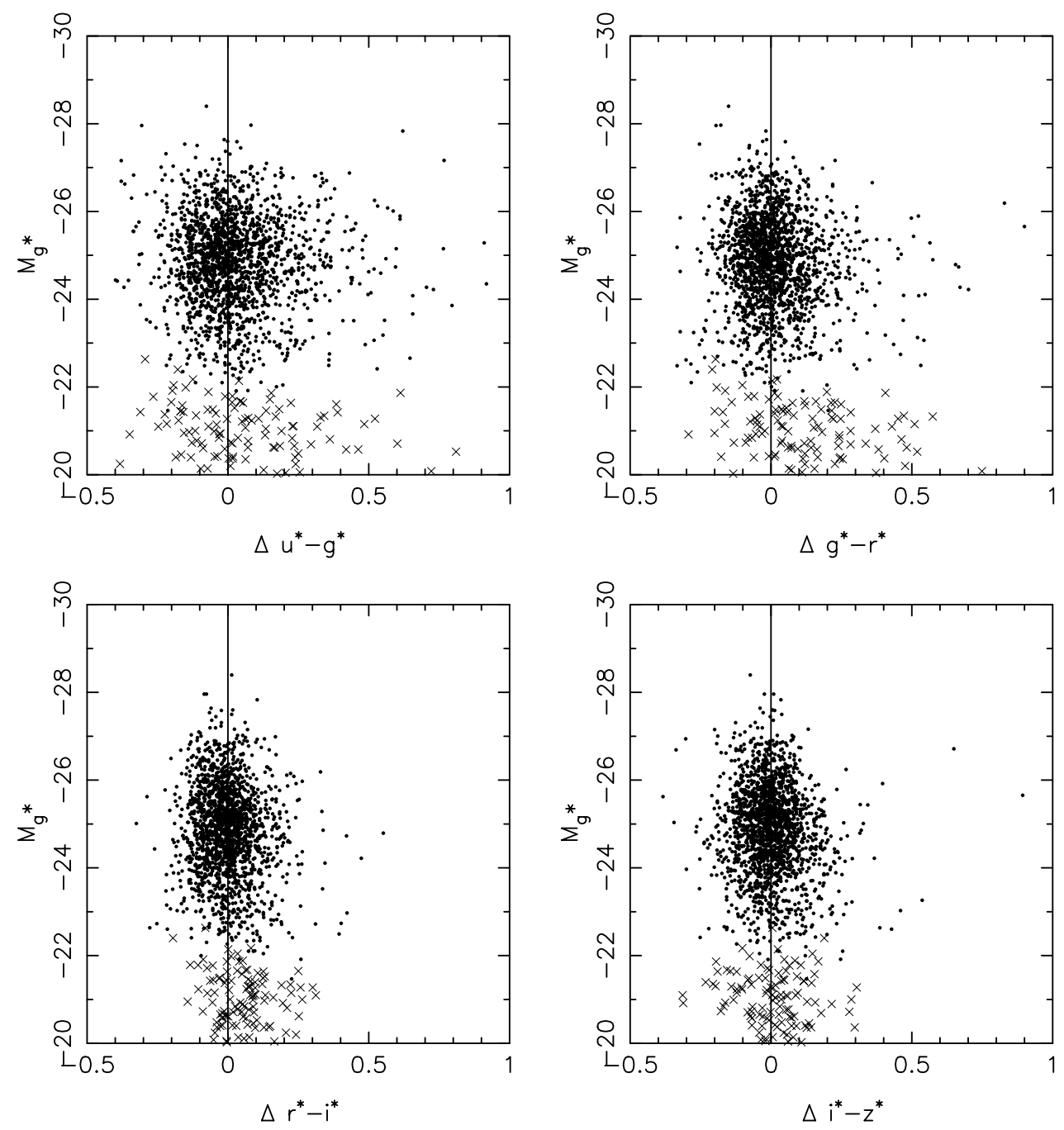

FIG. 11.-Quasar colors (corrected by the median colors as a function of redshift) plotted vs. absolute magnitude. Extended sources with $z \leq 0.4$ are plotted as crosses.

(2000). The blue extension of the contours would only be half as large if the observed effect was purely due to larger photometric errors at fainter magnitudes. The horizontal line in Figure 14 gives the $g^{*}$ magnitude for a limiting magnitude of $i^{*}=19$ assuming a power-law spectrum of $\alpha_{v}=$ -0.5 . The vertical line gives an approximate cutoff for UVX color selection (the actual SDSS color selection is done in three-dimensional color space and does not use this cut explicitly). To the limiting magnitude of the low-z SDSS quasar survey $\left(i^{*} \sim 19\right)$, the shifting of the stellar locus as a function of metallicity should have little effect upon the efficiency of quasar target selection at $z \leq 2.2$.

Futhermore, it is important to realize that the limiting magnitude of a sample is a function of the color of the sources. For example, note the decrease in faint, red sources in Figure 14. This dearth is a result of the fact that objects selected to $g^{*}=22$, must be bluer than $u^{*}-g^{*}=0.3$ in order to be brighter than the $u^{*}$ "plate limit" of $u^{*} \sim 22.3$. Thus a sample that includes objects as red as $u^{*}-g^{*}=0.8$ can only be considered to be complete to $g^{*}=21.5$.

\subsection{Extended Objects}

One of the many interesting questions that we can address with the SDSS quasar sample is whether quasars and Seyferts form a continuum or whether they represent physically distinct classes of objects. To address this issue it is necessary to target both point sources and extended sources when looking for quasars; the SDSS quasar target selection algorithm will not explicitly distinguish between quasars and Seyferts. However, it is equally important that we not target too many normal galaxies during the process of looking for quasars. As such, we must understand what regions of color space are populated by quasars with unresolved image profiles.

In our sample, extended source quasars are typically redder than point source quasars by $\sim 0.2$ in both $u^{*}-g^{*}$ and $g^{*}-r^{*}$. In $r^{*}-i^{*}$ and $i^{*}-z^{*}$ the colors of extended and point sources are more similar. That this is the case can be seen in Figure 5, where we plot as magenta points those $z \leq 0.6$ "quasars" that are flagged as extended objects in the SDSS database. The discrepancy in colors could have a number of origins, the most likely is that the extended sources are redder in the blue colors because of contamination from starlight from the quasar host galaxies. Such an effect could also be due to the strength of the small blue bump in low-redshift AGNs; we intend to investigate this possibility in future spectroscopic samples. Another possibility is that high-luminosity AGNs are more able to blow 

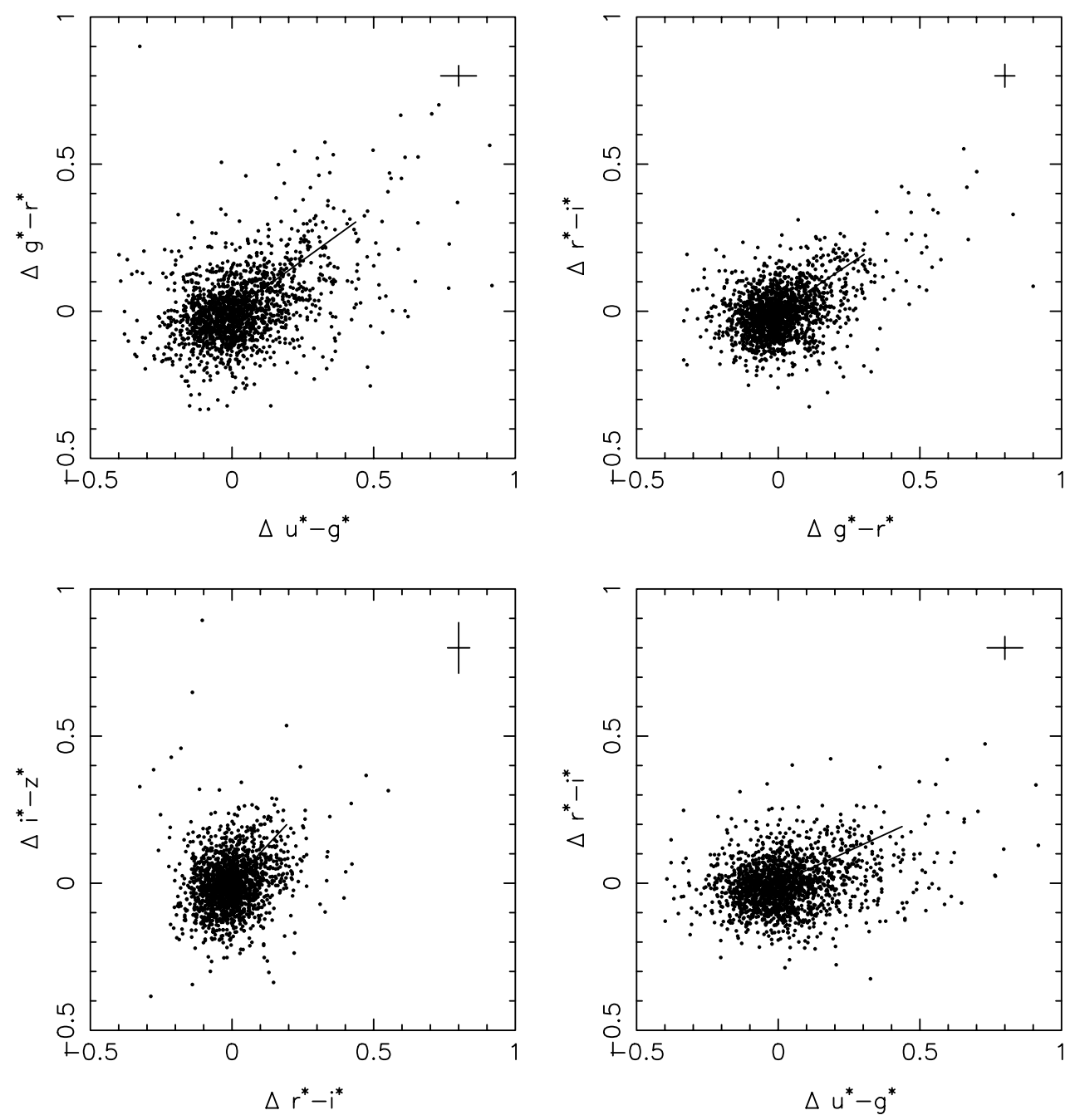

FIG. 12.-Quasar colors (corrected by the median colors as a function of redshift) plotted against each other. Typical errors are given in the top right-hand corner of each panel. The vector in each panel is the absolute value of the blue $95 \%$ confidence limit from Table 5 .

away dust in their vicinity and are therefore not as reddened. The SDSS sample will eventually cover enough area to assemble a large sample of high-luminosity lowredshift quasars, for comparison with the far more numerous low-luminosity low-redshift quasars. This will allow us to directly test the hypotheses of host galaxy contamination and luminosity- or redshift-dependent reddening.

We further investigate these possibilities by studying the colors of the objects in our sample as a function of absolute $g^{*}$ magnitude. Seyferts are typically defined as AGNs that are fainter than $M_{B}=-23$ and have broad emission lines. Whether or not this definition represents a distinction between two physically separate classes of objects is not at all clear (Schmidt \& Green 1983). Furthermore, Seyfert 2s, like Seyfert 1s have $M_{B}>-23$, but relatively narrow emission lines. It is an open question whether a population of "quasar $2 \mathrm{~s}$ " exists with space density relative to normal quasars comparable to the relative space density of Seyfert $2 \mathrm{~s}$ and Seyfert 1s. If not, this may represent a physical, luminosity-dependent difference between these classes of objects. Throughout, we refer to Seyfert 1s simply as Seyfert galaxies.

The problem with using $M_{B}=-23$ as the dividing line between quasars and Seyferts is that in any single survey with an optical flux limit, it is predominantly a division in redshift. At high redshift, the limiting magnitude of a sample causes a selection effect such that only quasars that are intrinsically bright are found. At low redshift, intrinsically faint objects are bright enough to make it into the sample; however, the volume of space sampled is so small that very few intrinsically bright objects are found. A selection criterion based on absolute magnitude is therefore essentially a redshift cut.

We test to see whether the traditional dividing line between quasars and Seyferts is appropriate and, if so, physical. If there exists a correlation between the absolute magnitude and the median-corrected colors, then it might be possible to use colors to discriminate quasars from Seyferts. We ask if there is any such evidence that the colors of quasars are a function of absolute magnitude.

The average absolute magnitude of quasars in our sample is a steep function of redshift for $z<1$, so we cannot simply ask if the colors of all $z<1$ quasars are a function of absolute magnitude. However, if we limit ourselves to small regions of redshift space, then we can assume that the average absolute magnitude is not changing across the (small) redshift bin. We have broken our low-redshift data set into redshift slices of $\Delta z=0.1$ from $z=0.1$ to $z=0.6$. 

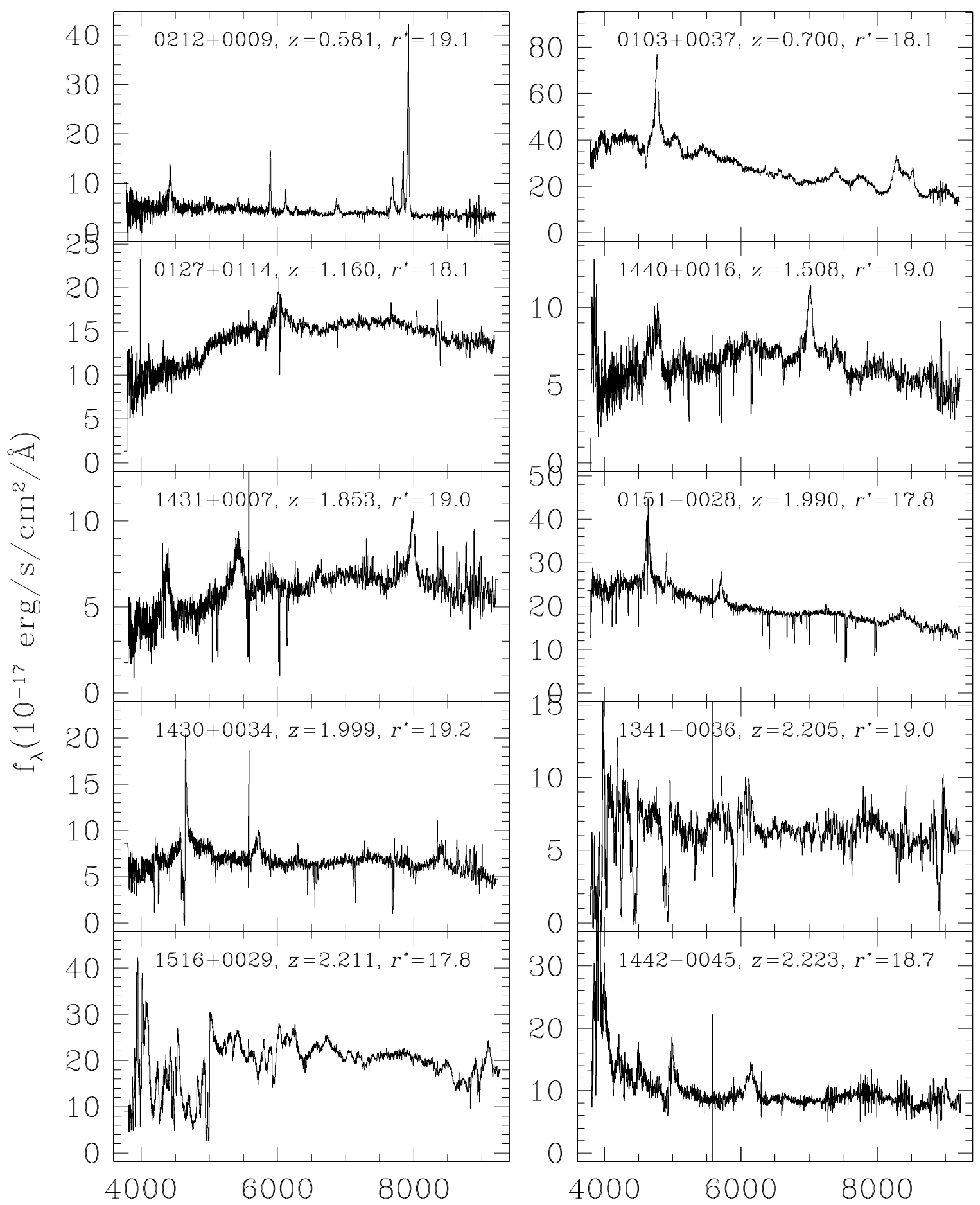

Wavelength $(\AA)$

FIG. 13. - Sample "red" quasars (SDSSp JHHMM \pm DDMM). That these quasars are anomalously red can be seen by comparing them with the more "normal" quasars at the same redshift shown in Fig. 1.

We find that within these redshift slices there is a correlation between the color and the absolute magnitude.

Since the average $M_{g *}$ changes significantly from bin to bin, we cannot simply make a plot of $M_{g *}$ versus color for the entire redshift range. Instead we have chosen to subtract the average $M_{g *}$ in each redshift bin and then stack the results for each of the six redshift slices. The result is Figure 15. Here we plot the residual color versus the normalized absolute magnitude for objects with $0.1 \leq z \leq 0.6$. If the colors of these objects were not a function of absolute magnitude, then the colors would be scattered around a color of zero. Instead we see that there is a strong correlation as a function of absolute magnitude in the sense that brighter objects (more negative normalized absolute magnitude) tend to be bluer. Furthermore, the redder objects have a tendency to be extended sources as might be expected if the 


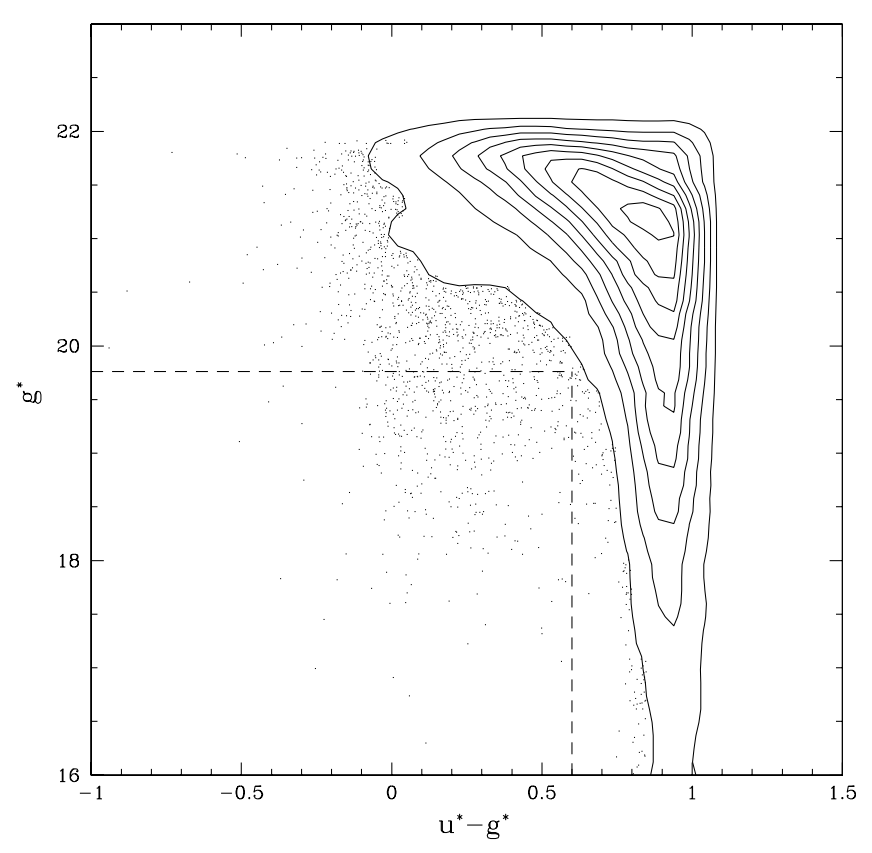

FIG. 14.- Stellar UVX sources. Stellar objects from camera column 3 of run 756 with $u^{*}-g^{*} \leq 1.0$ and $g^{*} \leq 22.0$. The steep fall-off of the red sources is artificial: it results from the red color-cut on the data. The vertical dashed lines shows an appropriate color-cut for UVX quasars in the SDSS system. The horizontal dashed line shows the average $g^{*}$ magnitude for a low-redshift quasar with $i^{*}=19.0$. Note the shifting of the stellar locus as a function of magnitude and the fall-off of faint, red sources.

fainter sources have a larger fraction of their light coming from the stars of the host galaxy. No such color-magnitude correlation is seen for a control sample of higher luminosity objects at higher redshift, again as expected if host galaxy contamination is the cause.

We conclude the following from Figure 15. Quasars and Seyfert galaxies form a continuum of properties. There is no apparent break in the color properties of quasars as function of absolute magnitude that would suggest a real physical difference between quasars and Seyfert galaxies. However, we do find that a cut in absolute magnitude at or near $M_{g *} \approx-23$ does exclude most of the extended sources from the quasar sample and that extended sources are significantly redder than point sources (in the blue colors). This distinction might be due to a physical difference in the strength of the $\lambda 3000$ bump between Seyferts and quasars, or due to the fact that the former have a larger contribution from their host galaxies. Arguing against the latter is the fact that the correlation between color and absolute magnitude holds for unresolved objects as well. More work is required to fully understand Figure 15 and what it says about the differences between low- and high-luminosity AGNs.

\section{CONCLUSIONS}

We have presented an empirical investigation of the colors of 2625 quasars $(0.04 \leq z \leq 5.28)$ in the SDSS photometric system. The number of quasars with accurate photometry that will result from the Sloan Digital Sky Survey will spur significant advances in quasar science in the coming years. The quality of the photometry allows for investigations that have hitherto been reserved for spectroscopic analysis. The combination of SDSS photometry and spectroscopy together will enable the resolution of a number of issues that currently perplex the quasar community. We have addressed some of those issues herein and have drawn the following conclusions.

1. Quasar colors vary significantly as a function of redshift, yet at a given redshift, the distribution of colors is surprisingly narrow. In four-dimensional color space, the colors of quasars are largely nondegenerate as a function of redshift. As a result, it may be possible to determine photometric redshifts for quasars, even at low redshifts.

2. Typical colors of quasars are consistent with a distribution of spectral indices centered on $\alpha=-0.5$ with a spread of \pm 0.65 ( $95 \%$ confidence). We find that the scatter in the colors of quasars at a given redshift must largely be due to a range of spectral indices as opposed to a range of emission line strengths, since the scatter is mostly independent of redshift for $z>0.5$. However, strong emission lines such as $\mathrm{Ly} \alpha, \mathrm{C}$ IV, $\mathrm{Mg}$ II, $\mathrm{H} \beta, \mathrm{H} \alpha$, and even the $\lambda 3000$ bump can have a significant affect upon the broadband colors of quasars. At low redshift, there is a larger scatter in the colors of quasars in the blue colors. This scatter may result from a wider range of strengths of the $\lambda 3000 \mathrm{bump}$ in lowredshift, low-luminosity quasars, and/or the low-redshift sample may be significantly contaminated by stellar light from the host galaxies.

3. Our sample shows no significant break in the colors of lower luminosity quasars (Seyfert galaxies) as compared with higher luminosity objects. However, we do find that lower luminosity quasars are redder than their higher luminosity counterparts for $0.05<z<0.65$. We find that the effect of using the traditional dividing line of $M_{B}=-23$ to separate the populations is to exclude all of the lowestredshift AGNs and most of those that are contaminated by stellar light from the host galaxy. However, it is unclear if this division is physical or simply observational.

4. Finally, we have discovered a statistical sample of reddened quasars. We find that the number of quasars with colors appreciably redder than the median is larger than might be expected in the absence of reddening. This reddening is probably not the result of stellar light from the host galaxy or absorption from intervening galaxies, since the reddening is not a function of redshift. Instead the observed reddening is probably internal to the quasars. Further examination of these quasars on an individual and on a group basis is in progress.

The Sloan Digital Sky Survey (SDSS) ${ }^{29}$ is a joint project of the University of Chicago, Fermilab, the Institute for Advanced Study, the Japan Participation Group, the Johns Hopkins University, the Max-Planck-Institute for Astronomy, New Mexico State University, Princeton University, the United States Naval Observatory, and the University of Washington. Apache Point Observatory, site of the SDSS, is operated by the Astrophysical Research Consortium (ARC). Funding for the project has been provided by the Alfred P. Sloan Foundation, the SDSS member institutions, the National Aeronautics and Space Administration, the National Science Foundation, the Department of Energy, Monbusho, and the Max Planck Society. The Hobby-Eberly Telescope (HET) is a joint project of the University of Texas at Austin, the Pennsylvania State

\footnotetext{
${ }^{29}$ At http://www.sdss.org/.
} 

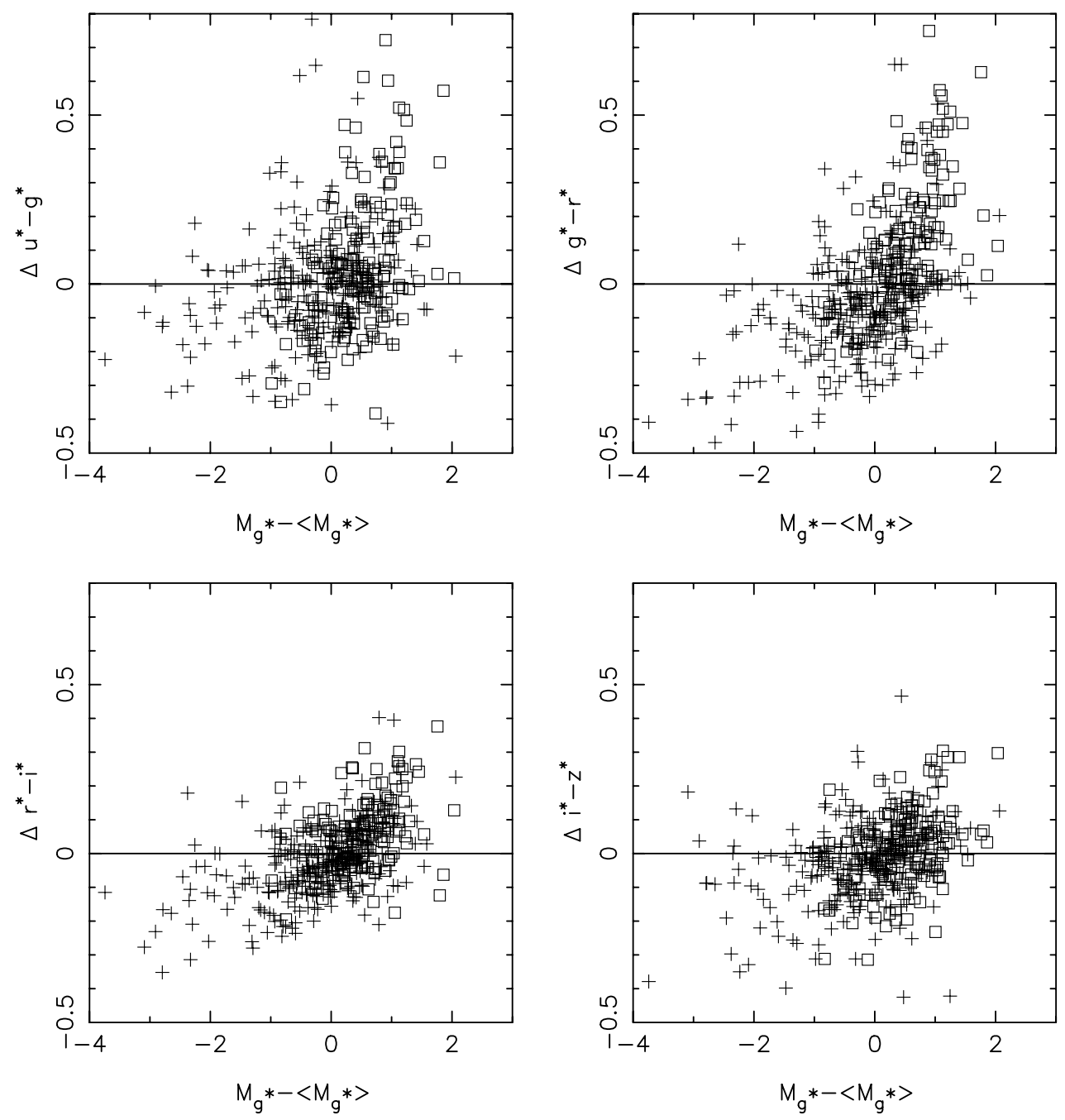

FIG. 15.-Quasar colors (corrected by the median colors as a function of redshift) plotted vs. normalized absolute magnitude. The absolute magnitude of the objects is normalized in redshift bins of $\Delta z=0.1$ from $z=0.1$ to $z=0.6$. Crosses are point sources, whereas squares represent extended sources. The lines show the median corrected color around which the points would be evenly distributed if there were not a correlation between color and luminosity. The mean absolute magnitude ranges from $M_{g *}=-20.1$ in the $z=0.1$ bin to $M_{g *}=-23.5$ in the $z=0.6$ bin.

University, Stanford University, Ludwig-MaximilliansUniversität München, and Georg-August-Universität Göttingen. The HET is named in honor of its principal benefactors, William P. Hobby and Robert E. Eberly. This research has made use of the NASA/IPAC Extragalactic Database (NED) which is operated by the Jet Propulsion Laboratory, California Institute of Technology, under contract with the National Aeronautics and Space Administration. D. P. S. and G. T. R. acknowledge support from
NSF grant AST 99-00703. X. F. and M. A. S. acknowledge support from Research Corporation, NSF grants AST 9616901 and AST-00 71091, the Princeton University Research Board, and a Porter O. Jacobus Fellowship. We thank Sofia Kirhakos and Emily Laubacher for their assistance in an early stage of this project. We thank Bob Becker for providing data in advance of publication. We thank Pat Hall and David Weinberg for critical readings of the manuscript and for the resulting improvements.

\section{REFERENCES}

Annis, J. A., et al. 2001, in preparation

Becker, R. H., et al. 1998, BAAS, 192, 1101 2001 , in preparation

Becker, R. H., White, R. L., \& Helfand, D. J. 1995, ApJ, 450, 559

Boyle, B. J., Fong, R., Shanks, T., \& Peterson, B. A. 1990, MNRAS, 243, 1

Boyle, B. J., Shanks, T., \& Peterson, B. A. 1988, MNRAS, 235, 935

Brandt, W. N., et al. 2000, AJ, 119, 2349

Budavári, T., Szalay, A. S., Connolly, A. J., Csabai, I., \& Dickinson, M. 2000, AJ, 120, 1588

Crampton, D., Janson, T., Durrell, P., Cowley, A. P., \& Schmidtke, P. C. 1988, AJ, 96, 816

Doi, M., et al. 2001, in preparation

Fall, S. M., \& Pei, Y. C. 1993, ApJ, 402, 479

Fan, X. 1999, AJ, 117, 2528

Fan, X., \& Chen, J. 1994, Chinese Astron. Astrophys., 277, L5
Fan, X., et al. 1999, AJ, 118, 1
. 2000, AJ, 119, 1 2000, AJ, 119, 1

Finlator, K., et al. 2000, AJ, 120, 2615

Francis, P. J. 1996, Publ. Astron. Soc. Australia, 13, 212

Francis, P. J., Hewett, P. C., Foltz, C. B., Chaffee, F. H., Weymann, R. J., \& Morris, S. L. 1991, ApJ, 373, 465

Francis, P., Whiting, M., \& Webster, R. 1999, BAAS, 194, 11301

Frieman, J., et al. 2001, in preparation

Fukugita, M., Ichikawa, T., Gunn, J. E., Doi, M., Shimasaku, K., \& Schneider, D. P. 1996, AJ, 111, 1748

Grandi, S. A. 1982, ApJ, 255, 25

Green, R. F., Schmidt, M., \& Liebert, J. 1986, ApJS, 61, 305

Gregg, M. D., Becker, R. H., White, R. L., Helfand, D. J., McMahon, R. G., \& Hook, I. M. 1996, AJ, 112, 407 
Gunn, J. E., et al. 1998, AJ, 116, 3040

Hawkins, M. R. S., \& Veron, P. 1993, MNRAS, 260, 202

Hewett, P. C., Foltz, C. B., \& Chaffee, F. H. 1995, AJ, 109, 1498

Hewitt, A., \& Burbidge, G. 1989, ApJS, 69, 1 (HB89)

Irwin, M., McMahon, R. G., \& Hazard, C. 1991, in ASP Conf. Ser. 21, The Space Distribution of Quasars, ed. D. Crampton (San Francisco: ASP), 117

Kent, S., et al. 2001, in preparation

Lupton, R. H., Gunn, J. E., \& Szalay, A. S. 1999, AJ, 118, 1406

Lupton, R. H., et al. 2001, in preparation

Mathis, J. S. 1994, ApJ, 422, 176

Mushotzky, R. F., Cowie, L. L., Barger, A. J., \& Arnaud, K. A. 2000, Nature, 404, 459

Netzer, H., Wamsteker, W., Wills, B. J., \& Wills, D. 1985, ApJ, 292, 143

Newberg, H. J., Richards, G. T., Richmond, M., \& Fan, X. 1999, ApJS, 123, 377

Newberg, H., et al. 2001, in preparation

Oke, J. B., \& Gunn, J. E. 1983, ApJ, 266, 713

Oke, J. B., Shields, G. A., \& Korycansky, D. G. 1984, ApJ, 277, 64

Peterson, B. M., ed. 1997, An Introduction to Active Galactic Nuclei (Cambridge: Cambridge Univ. Press)

Petravick, D., et al. 1994, Proc. SPIE, 2198, 935

Pier, J., et al. 2001, in preparation

Richards, G. T., et al. 2000, BAAS, 196, 5304

Richards, G. T., et al. 2001, in preparation

Richstone, D. O., \& Schmidt, M. 1980, ApJ, 235, 361

Sandage, A. 1965, ApJ, 141, 1560
Schlegel, D. J., Finkbeiner, D. P., \& Davis, M. 1998, ApJ, 500, 525

Schmidt, M. \& Green, R. F. 1983, ApJ, 269, 352

Schneider, D. P., Schmidt, M., \& Gunn, J. E. 1994, AJ, 107, 1245 - 1999, AJ, 117, 40

Schneider, D., et al. 2001, AJ, 121, 1232

Schneider, D. P., et al. 2000, PASP, 112, 6

Siegmund, W., et al. 2001, in preparation

Smith, A., et al. 2001, AJ, submitted

Spinrad, H., Marr, J., Aguilar, L., \& Djorgovski, S. 1985, PASP, 97, 932

Stepanian, J. A., Lipovetsky, V. A., Chavushian, V. H., Erastova, L. K., \&

Balayan, S. K. 1993, Bull. Special Astrophys. Obs., 36, 5

Szalay, A., et al. 2001, in preparation

Tucker, D., et al. 2001, in preparation

Uomoto, A., et al. 2001a, in preparation

. 2001b, in preparation

Vanden Berk, D. E., et al. 2000, BAAS, 196, 5003 2001, AJ, submitted

Véron-Cetty, M. \& Véron, P. 1996, A Catalogue of Quasars and Active Nuclei (7th ed.; Garching: ESO)

Warren, S. J., Hewett, P. C., Irwin, M. J., \& Osmer, P. S. 1991a, ApJS, 76, 1

Warren, S. J., Hewett, P. C., \& Osmer, P. S. 1991b, ApJS, 76, 23 1994, ApJ, 421, 412

White, R. L., et al. 2000, ApJS, 126, 133

Wolf, C., Meisenheimer, K., \& Röser, H.-J. 2000, A\&A, 365, 660

Yanny, B., et al. 2001, in preparation

York, D. G., et al. 2000, AJ, 120,1579

Zheng, W., et al. 2000, AJ, 120, 1607 